\title{
Le corps et son anatomie. Représentations et croyances
}

Enquêtes auprès d'étudiants en staps

\section{Marie-Joseph Biache}

\section{(2) OpenEdition}

\section{Journals}

Édition électronique

URL : https://journals.openedition.org/tc/161

DOI : $10.4000 /$ tc. 161

ISSN : 1952-420X

\section{Éditeur}

Éditions de l'EHESS

Édition imprimée

Date de publication : 1 juin 2002

ISSN : 0248-6016

\section{Référence électronique}

Marie-Joseph Biache, «Le corps et son anatomie. Représentations et croyances », Techniques \&

Culture [En ligne], 39 | 2002, mis en ligne le 12 juin 2006, consulté le 29 septembre 2022. URL : http:// journals.openedition.org/tc/161; DOI : https://doi.org/10.4000/tc.161

Ce document a été généré automatiquement le 29 septembre 2022.

Tous droits réservés 


\title{
Le corps et son anatomie. Représentations et croyances
}

\author{
Enquêtes auprès d'étudiants en staps ${ }^{1}$
}

\author{
Marie-Joseph Biache
}

1 Lorsque l'on questionne les bacheliers candidats à l'entrée en DEUG de STAPS (Sciences et techniques des activités physiques et sportives), l'enseignement de l'anatomie humaine est systématiquement évoqué à la fois comme nouveau, en rupture avec la formation secondaire suivie, et absolument indispensable. La raison avancée est avant tout utilitaire : la connaissance de l'anatomie se présente comme un fondement obligé de l'exercice des métiers de l'éducation physique et du sport ${ }^{2}$. Elle répond à une des aspirations majeures qu'expriment les étudiants débutants, celle de la connaissance de leur corps, mais représente parallèlement pour eux la particularité des études universitaires, à savoir l'accès à l'analyse rationnelle des activités sportives, dont ils n'ont à ce moment qu'une expérience pratique, subjective et dont la connaissance se réduit à un ensemble de conseils, injonctions ou règles, réunis dans le discours technique particulier de leur sport habituel.

2 L'accès au cursus universitaire est donc marqué par la contradiction entre une attente d'objectivation, de connaissance explicite du corps humain fondée sur sa matérialité, sa configuration comme sa constitution fonctionnelle, et un désir subjectif de compréhension de soi ainsi que de justification des usages du corps propre.

L'anatomie du corps humain : matérialité et réalité

3 L'enseignement de l'anatomie participe de la formation scientifique des étudiants en STAPS. Il s'agit de l'anatomie macroscopique, celle des organes à l'état cadavérique. On peut s'étonner de cette persistance de la référence à l'anatomie macroscopique dans des études non médicales, alors que celle-ci est un "phénomène achevé » qui s'est développé durant cinq siècles en Europe "selon deux voies, celle de l'anatomie pour artistes et celle de l'anatomie pour médecins » (Descargues $1980: 8$ ).

4 C'est la réalité du corps humain qui est posée, nommée dans ses parties (anatomie descriptive), normée dans les articulations entre ces parties (anatomie fonctionnelle). 
Mais cette réalité est réduite, puisque les enseignements portent principalement sur l'appareil locomoteur (les muscles striés et le squelette osseux), choix qui exclut les viscères $-\mathrm{y}$ compris le cœur- et la tête.

5 L'anatomie est définie comme science fondamentale car elle promeut un matérialisme ontologique et évacue apparemment tout questionnement psychologique ou philosophique. Elle décrit et analyse un corps non métaphysique, un corps désubjectivé, mobile segmenté soumis aux lois d'une physique mécanique selon laquelle l'espace et le temps prennent valeur de "réalité supérieure » à celle des sensations (Mach 1984).

6 La connaissance de cette réalité physique, «le corps humain ", devrait donc constituer la base rationnelle de l'entraînement sportif tel qu'un enseignement universitaire l'envisage. La rationalisation du mouvement s'effectue sur le mode de la synthèse entre la structure anatomique du corps humain et les lois de la mécanique classique du mouvement des solides (cinématique) enseignée par ailleurs. Dans ce cas, la connaissance anatomique du corps reste première et affirmée comme nécessaire à toute étude du mouvement visant l'apprentissage et la mise en œuvre dans le cadre d'une pédagogie sportive ${ }^{3}$. Cette conception est proprement physicaliste. Le corps est envisagé comme étendue mobile et l'étude positive du mouvement revient à l'analyse de la complexion matérielle du corps ainsi que de la nature des trajectoires, segmentaires ou globales. Ernst Mach, dans ses Remarques préliminaires antimétaphysiques (1984), souligne l'illusion que constitue la conception physicaliste d'un corps stable. Pour cet auteur, le corps humain, qu'il assimile au moi, ne forme pas une réalité matérielle, mais un complexe de sensations. Son unité n'est pas substantielle; elle est ou bien métaphysique -le corps en soi projeté dans sa matérialité supposée- et se révèle une erreur intellectuelle, ou bien sensorielle : un complexe de sensations cimentées en une entité pratique.

7 Le corps humain présente ainsi une ambiguïté fondamentale : en tant que matière, il se prête à une définition physique, mais cette définition n'est pas incluse dans la description anatomique; en tant que complexe sensoriel (ou perceptif), il est fondamentalement lié à l'unité pratique du moi.

8 L'enseignement de l'anatomie se heurte donc à un obstacle qui est le corps humain luimême. Puisque le point de vue promu n'est pas celui d'une intervention directe sur la matière corporelle ${ }^{4}$-ce qui est le cas en chirurgie ou en médecine-, et puisque la justification pour les études en STAPS est celle de l'analyse du mouvement à des fins pédagogiques, le corps ne peut échapper à la recomposition psychologique, à la synthèse pratique. La réalité est par conséquent phénoménale et n'exclut ni les représentations psychiques, ni les interprétations métaphysiques. Le réalisme anatomique dépasse l'unité matérielle du corps humain. "Le corps est un et inchangeable aussi longtemps que nous n'avons pas besoin de prendre en considération ses particularités » (Mach 1984 :107).

9 Or, l'anatomie enseignée en STAPS est doublement particulière : par sa prétention à se poser comme cause physique du mouvement, et par son optique, sa focalisation sur les muscles striés et les os. Loin de constituer une description froide de la structure physique du corps, elle impose, par ce qu'elle suppose, la composition de théories du corps par les étudiants, mais ne l'éclaircit pas épistémologiquement.

Les étudiants sont donc amenés à réaliser l'anatomie du corps humain dans un conflit entre une anatomie académique sue (qui apparaît comme connaissance d'objet) et une 
anatomie corporelle éprouvée, qui se traduit dans des représentations du corps sur le mode assertorique de la croyance.

Le contexte de l'étudePopulation

11 L'étude a porté sur les représentations de l'anatomie chez les étudiants ayant suivi les cours d'anatomie descriptive et fonctionnelle inscrits dans le cursus du DEUG de STAPS.

Les résultats sont issus de seize entretiens de longue durée effectués en deux phases. La première série d'entrevues s'est déroulée en 1991, auprès de sept étudiants, dont quatre jeunes femmes, la seconde en 2001, auprès de neuf étudiants, dont cinq jeunes femmes.

13 Tous ces informateurs sont, au moment des entretiens, des acteurs du sport. Leurs niveaux de pratiques dans diverses activités s'échelonnent entre les niveaux régional, interrégional, national et international. Par ailleurs, ils sont en charge, pour la plupart, dans leur spécialité, de la formation des jeunes compétiteurs ou de l'initiation sportive des enfants. Les étudiants interrogés étaient volontaires et s'attendaient à pouvoir développer leur avis sur le contenu des études STAPS, thème affiché des entretiens, sans précision particulière concernant les enseignements d'anatomie.

14 Il faut souligner qu'entre 1991 et 2001, les contenus des cours d'anatomie humaine n'ont pas varié: il s'agit principalement, comme nous l'avons déjà signalé, de la description du squelette osseux et de l'appareil musculaire. En revanche, l'enseignant en charge de ces cours a changé, et avec lui la pédagogie. En 1991, le cours magistral était exposé en recourant parallèlement à la projection de schémas anatomiques, à un squelette humain véritable, ainsi qu'à la reproduction d'un corps écorché. En 2001, le support visuel est principalement constitué de schémas projetés qui sont des figures simplifiées de préparations anatomiques sur cadavres.

15 Ce changement a eu des conséquences sur les propos des étudiants interrogés. Et cela, surtout en termes de références: les entretiens de 2001 évoquent plus fréquemment l'appareil musculaire que le système osseux, celui-ci semblant en quelque sorte être subordonné au premier dans leur référence à l'anatomie. Les étudiants de 1991, eux, s'appuient plutôt sur la structure du squelette pour illustrer leurs représentations de l'anatomie humaine.

Méthodologie de l'entretien

Chaque entretien a suivi son cheminement propre sans toutefois qu'y soit omise l'évocation de trois thèmes.

17 1) Intérêt de l'anatomie en fonction des études suivies et des professions envisagées. Il s'agit essentiellement de saisir comment l'étudiant relie les contenus des cours à ses pratiques quotidiennes d'acteur du sport, et cela face à la justification rationnelle de ces contenus, telle qu'elle se formule à l'origine du recours à l'anatomie comme science d'appui pour une formation universitaire dans les activités physiques et sportives.

18 2) Le rapport au corps et la nature de ses représentations au travers de la connaissance anatomique acquise. Les étudiants visent une double formation qui peut sembler contradictoire. En effet, tout en accédant à des connaissances objectives sur la structure matérielle du corps humain, ils élaborent, par la multiplication des expériences sportives techniquement interrogées, un savoir subjectif concernant leur corps propre.

19 3) La matérialité du corps humain et sa connaissance objective. On peut avancer l'hypothèse d'un recours à la dissection pour une connaissance plus objective. Celle-ci 
est de même nature que l'appui sur un squelette véritable. Fondamentalement, il s'agit dans cette optique de considérer le corps humain sous l'angle de son organisation matérielle, ce qui est une des raisons alléguées pour justifier l'existence des cours d'anatomie en STAPS. La structure matérielle du corps peut constituer une cause première du mouvement et son étude paraît donc nécessaire, mais devient dans ce cas une science du cadavre.

Ces trois grands thèmes ont trouvé des déclinaisons particulières selon les étudiants interviewés. Chaque entretien se terminait par la présentation de huit reproductions (voir figures en annexe), tirées d'ouvrages d'anatomie humaine, et nous demandions à l'étudiant de commenter ces images selon deux critères de jugement : leur conformité avec sa conception de l'anatomie, et son inclination à les retenir ou à les rejeter spontanément ${ }^{5}$.

Présentation des planches anatomiques

21 Les huit planches anatomiques présentées aux étudiants ont été retenues selon les couples d'opposition suivants :

- réalité matérielle/figuration sublimante;

- précision/indistinction;

- figuration partielle, locale/reproduction globale du corps;

- image du mouvement/corps inanimé;

- présence de la peau/corps écorché;

- focalisation sur les muscles/vision des « organes »;

- homme/femme;

- figuration ancienne/photographie ou dessin contemporain.

Les numéros attribués aux différentes planches l'ont été par hasard et ont pour fonction essentielle de permettre un repérage des commentaires. La présentation des planches aux informateurs est groupée, les planches étant distribuées aléatoirement dans le paquet présenté.

Analyse des entretiensL'intérêt de l'anatomie, l'intérêt pour l'anatomieUn savoir requis «L'anatomie est indispensable, il faut qu'on ait des notions pour ce qu'on veut faire.

Il faut des notions sur les muscles pour la vie de tous les jours. » (Élodie, J. 2001)

La nécessité d'une connaissance de l'anatomie du corps humain est conçue d'emblée par les étudiants, mais elle est posée comme relative. Si une connaissance globale, en quelque sorte utilitaire, est recherchée, attendue, elle n'est en aucune façon entendue sur le mode exhaustif.

Dès le départ, le savoir anatomique est frappé d'ambiguïté : son intérêt oscille entre la recherche d'une connaissance instrumentale et celle d'une connaissance objective, parée des valeurs de la positivité scientifique.

«L'anatomie, c'est la connaissance de l'ostéologie et de la myologie. On doit aussi voir l'action des muscles dans la marche et la course. C'est un peu une découverte du corps humain. » (Marie-Aude, L. 1991)

Un savoir utile

Connaître le corps humain est une obligation pour qui se destine à la pédagogie sportive ou à la performance. Le corps est l'outil premier et la performance n'est jamais séparable du corps même. L'anatomie permet de passer d'un corps vu à un corps su. 

performance que le savoir anatomique est bien souvent considéré comme le plus utile. L'intérêt pour le corps ne se réduit pas à une simple curiosité, mais s'inscrit dans l'idée de prescriptions des exercices, d'un gouvernement possible des entraînements. Dans cette optique, le corps est recomposé : l'utilité de l'anatomie est locale, circonscrite à tel ou tel groupe musculaire particulièrement sollicité lors d'un acte.

«Ce qui serait intéressant, c'est de faire le lien entre la forme, la longueur du muscle et son utilité... pour moi, l'anatomie, on s'en sert. » (Stéphanie, D. 2001)

Le regard n'est pas annulé, il reste premier dans toute pratique. L'utilité accordée aux connaissances anatomiques réside dans le renforcement de ce regard, son armement par un savoir, la restriction rationnelle de son champ.

"Ça peut aider à comprendre certaines postures qu'il faut prendre pour faire certains gestes; enfin moi, je l'ai retrouvé en athlétisme par exemple... j'avais fait de l'athlétisme l'an dernier, et là, cette année j'ai fait donc le bassin, la jambe, on a parlé du quadriceps, tout ça, son importance dans... et ça m'a fait tilt, ça fait repenser... d'un côté c'est plus sérieux, je crois. » (Hervé, L. 1991).

Apparait donc l'obligation d'une représentation mentale qui n'est pas l'unique image saisie du mouvement mais sa perception organisée. Le savoir anato-mique acère le regard porté sur le corps performant. L'utilité pratique des connaissances anatomiques est systématiquement reliée à trois domaines : la santé et la prévention des accidents, l'entraînement et l'amélioration de la performance, la connaissance et la maîtrise du mouvement.

«L'anatomie, c'est intéressant... c'est important s'il y a des problèmes, savoir si c'est au niveau musculaire, au niveau ligamentaire... même pour les professeurs d'EPS, je pense c'est intéressant d'en savoir un minimum. » (Fabien, C. 2001)

Dans ce cas, la connaissance anatomique est censée participer à l'organisation de l'entraînement, des charges de travail, en fournissant la possibilité d'un diagnostic. Il reste que son utilité, bien qu'affirmée, est relativisée, et l'explication fournie par l'informateur, lui-même hockeyeur sur glace de haut niveau, exprime plus un désir qu'une affirmation.

"Ça peut être obligatoire, surtout s'il y a un problème sur la glace ou dans n'importe quel sport s'il y a un enfant qui se fait une contracture musculaire, on le connaît en physio comment ça se passe, mais au niveau du muscle ... que ce soit tel ou tel muscle, ça devrait apporter quelque chose... » (Fabien, C. 2001)

l'anatomie. Les informateurs qui developpent ce theme font tous reference à la connaissance du rachis. La gravité des blessures possibles de la colonne vertébrale est soulignée; elle est reliée à la complexité de la structure vertébrale elle-même, mais aussi aux conséquences motrices que de telles blessures pourraient avoir.

«Je sais que tout ce qui est lombo-sacré, c'est important. Moi, j'ai vu souvent et même j'ai vu, quand moi j'étais à l'école, des chutes... où on relevait les gens comme ça, quoi, sans essayer de prendre quelques précautions pour voir s'il n'y avait pas eu de problème au niveau cervical, dorsal, etc. On sait suivant quel signe moteur s'il y a eu dans toute la colonne vertébrale un choc, une lésion, etc. " (Nathalie, F. 1991) ${ }^{6}$

Prévenir les accidents est malgré tout un souci second, tant c'est à l'amélioration de la muscle et son utilte... pour moi, lanatomie, on s'en sert. » (Stéphanie, D. 2001)

La différence déjà signalée entre les étudiants de 1991, lesquels ont une vue plus globale du corps, plus architecturale, et ceux de 2001, qui divisent le corps en structures locales perçues à partir de la disposition des muscles, est particulièrement marquée sur ce point. L'évolution du niveau de performance recherché correspond à un changement 
de regard porté sur le corps : le savoir anatomique devient un outil d'analyse. Il permet de se placer au plus près du muscle.

«Au niveau des entraîneurs, à un haut niveau, c'est vital... il faut en tenir compte pour la construction d'une performance et pour la préparation physique, savoir quel muscle travaille et à partir de là voir l'orientation des fibres pour pouvoir les renforcer. » (Cyril, C. 2001)

Entraîner revient à régler au mieux le corps pour qu'il reproduise une performance, donc à s'approcher au plus près des éléments moteurs.

La connaissance anatomique semble par conséquent constituer une aide précieuse pour la composition des exercices et s'impose comme base nécessaire moins pour la conception même de l'entraînement que pour l'ajustement des mouvements. Composer un mouvement, en accroître l'efficience trouve dans l'anatomie sa justification, mais non sa raison première. Se fait ainsi jour une interversion : ce n'est pas la connaissance anatomique qui permet l'élaboration des exercices d'entraînement; ce sont au contraire ceux-ci qui valident la nécessité de cette connaissance. La technique reste première par rapport à son fondement physique, mais elle est légitimée par l'analyse descriptive de celui-ci. L'étude de l'organisation physique du corps est conçue comme utile et nécessaire par les étudiants, mais son importance est moins épistémique que pratique.

« Si on n'a pas la connaissance de ses muscles, on ne sait pas décomposer vraiment le mouvement; et puis je pense que si on sait quel muscle on doit actionner pour agir... en fait de savoir les noms, bon, c'est le principe, c'est pas vraiment nécessaire, mais de connaître les muscles, je pense que ça l'est... » (Rodolphe, B. 1991)

Pourtant, le savoir anatomique est avant tout perçu comme science du corps humain et présente aux yeux des étudiants des valeurs de rationalité dont les pratiques d'entraînement ne bénéficient pas.

Un savoir rigoureux

35 C'est un réalisme naïf que traduit à un premier niveau de réponse la valeur positive attribuée à l'anatomie: le corps humain apparaît comme une réalité indiscutable, concrète, qui ne suppose pas de réflexions ou de discussions particulières.

«L'anatomie, ça permet l'étude du mouvement, une analyse du mouvement plus basique, moins philosophique. » (Cyril, R. 2001)

« Moi, je suis encore assez terre-à-terre et j'ai plutôt l'esprit scientifique. J'aime bien les sciences pures, tout ce qui est anatomie, biologie, physiologie, c'est du concret pour moi. » (Audrey, B. 2001)

«Pour moi, l'anatomie, c'est concret, c'est la réalité. C'est des choses qu'on peut pas remettre en cause. » (Stéphanie, D. 2001)

Décrire le corps humain, c'est adopter un esprit scientifique tout entier inclus dans un matérialisme apparent. C'est quitter le plan du débat métaphysique incertain et se placer dans celui d'une réalité irrécusable. La description anatomique est posée comme science par les étudiants et s'inscrit à ce titre dans le programme des études. Sa qualité de science repose sur deux critères.

Le premier critère est son objet, le corps humain, qui bénéficie d'une double valeur de réalité. Il est d'abord réalité physique descriptible; il est aussi réalité pour soi, réalité d'existence, le corps propre. La réalité qui donne au savoir anatomique sa valeur positive est une élaboration syncrétique qui mêle réalisme d'objet et réalisme du sujet. 

qu'on l'a découpé ». Il se présente comme matérialité ultime; la rationalité de son savoir anatomique est entièrement inscrite dans cette matérialité et la méthode qui permet d'y accéder. Si l'anatomie est « infaillible », si elle peut se parer de l'éclat de la vérité, c'est parce qu'elle place dans l'image les caractères topographiques de l'étendue qu'elle décrit. Cet effort d'exactitude n'est toutefois pas dépendant de la seule matérialité du corps. Le corps étant une étendue substantielle il exige un effort de repérage. Le savoir anatomique, infailliblement constitué à l'aide de dissections des corps, oblige à une recomposition exacte des parties de ces corps en un paysage corporel global organisé dans l'espace. Si l'anatomie est une science, c'est qu'elle contraint à une telle recomposition et que celle-ci concerne le corps propre. La subjectivité se réfère à l'objectivité, qui seule est susceptible de produire des repères adéquats, des réseaux logiques non contaminés par des constructions sensibles.

«Il faut que ça soit un mode de compréhension de soi, un des modes de compréhension de soi, de ses comportements, et... de ses mouvements plus exactement, plus que... comportement, c'est beaucoup plus psychologique que ça, non, c'est plutôt les mouvements, pour expliquer les mouvements... de soi, oui !» (Pascal, B. 1991)

Chez certains informateurs, la logique du corps est référée à une conception morphogénétique ou plus simplement à un déterminisme de forme. Ainsi la qualité de rigueur qui marque le savoir anatomique est tout simplement due à la nature du corps elle-même. Il ne s'agit plus de son unique matérialité, mais des lois d'évolution qui y ont mené. Le savoir anatomique ne se suffit donc plus à lui-même, puisque outre la structure connue, il doit également englober la fonction qui, dans le cas des études en STAPS, est ramenée au mouvement corporel.

«Il serait plus intéressant de savoir, pour tel muscle, comment il fonctionne et pourquoi il fonctionne comme ça, alors que là, on apprend que le muscle est à tel 
endroit... enfin... s'il est là, c'est quand même qu'il a une utilité, sinon il serait pas

là. » (Stéphanie, D. 2001)

L'exactitude change de plan : tantôt placée dans la topographie précise de la matérialité du corps, tantôt rapportée à un schème de composition spatiale, elle peut également s'appliquer à la compréhension du rôle fonctionnel -ramené aux mouvements- de la structure ostéo-musculaire du corps.

Il en découle une indistinction, une variabilité de la représentation du corps anatomique : celui-ci acquiert parfois une essence hybride, celle d'un composé physicobiologique; il est à la fois réalité mécanique et réalité biologique.

«L'anatomie, c'est l'explication mécanique de la mécanique du corps.» (Rodolphe,

B. 1991)

«L'anatomie oublie la tête et donc ça veut bien dire que l'anatomie c'est une science qui fait abstraction de tout ce que l'homme peut ressentir; c'est très mécanique, c'est pour ça que pour moi, c'est plus de la mécanique qu'autre chose l'anatomie. Ça s'arrête là ! Ça sert à comprendre mais ça ne va pas plus loin.» (Rodolphe, B. 1991) mouvement ne sont pas inclus dans cette représentation de l'anatomie. Le corps est une machine soumise aux lois de la mécanique classique. Il faut ramener l'anatomie à l'étude mécanique du corps humain sur le modèle de la iatromécanique de Borelli. Mais un tel point de vue est insuffisant car le mouvement n'est pas que la mobilité. Il suppose une fonction autre que la seule application des lois de la mécanique ${ }^{8}$. La " vie intérieure » évoquée est proprement anatomique pour Nadia L. même si l'anatomie ne la recouvre pas totalement.

«La vie intérieure, c'est plus fort encore. Ce qu'on apprend en anatomie, c'est superficiel. » (Nadia, L. 1991)

Le corps tel qu'elle le définit reste une machine, non pas uniquement par analogie des composants, mais en tant que moyen de produire des mouvements.

«Oui, oui... c'est une machine. Descartes dit que avant que l'être soit esprit, il est avant tout une machine d'os et de chair. » (Nadia, L. 1991)

L'exactitude du savoir anatomique n'est plus alors assimilée à la seule précision de l'analyse des structures, ni même dans les lois de la mobilité d'un assemblage, d'une configuration, mais dans l'exigence d'une «vie intérieure » à l'origine du mouvement spontané. Il faut malgré tout remarquer que la référence à une anatomie mécanique provient des informateurs interrogés en 1991. Ceux qui ont été questionnés en 2001 ont plutôt tendance à opposer anatomie et mécanique. Ainsi à propos de la planche 7.

"Ça fait plutôt biomécanique qu'anatomique. La biomécanique c'est plus une étude du mouvement en fonction des différents segments du corps. » (Fabien, C. 2001)

48 La connaissance anatomique est dans ce cas d'abord reliée à la configuration ostéomusculaire du corps, cette dernière apparaissant comme cause possible du mouvement. Toujours à propos de la planche 7 :

«On voit pas vraiment les muscles, c'est plus de la biomécanique avec les mouvements des os et tout ça... Là, il y a pas de muscles : il y a vraiment l'angulation des différents membres pour effectuer un mouvement mais là on voit pas les muscles. » (Audrey, B. 2001)

Cette différence semble traduire le passage d'une iatromécanique avant tout centrée sur la configuration d'un dispositif -les cours d'anatomie étaient illustrés par un squelette véritable en 1991 - à une mécanique fonctionnelle ${ }^{9}$ dans laquelle la cause du 
mouvement est attribué aux muscles, et qui correspond à un enseignement contemporain essentiellement fondé sur des schémas. Ce n'est plus alors un mécanisme de structure qui s'impose, appuyé sur la nature de la configuration de la machine, mais un mécanisme de fonction qui concerne la cause efficiente du mouvement. Dans les deux cas néanmoins, l'origine du mouvement est imputée à l'anatomie, en laquelle cause matérielle et cause efficiente sont confondues.

L'aspiration des étudiants est d'accéder, par le savoir anatomique, à la possibilité d'une analyse rigoureuse du mouvement corporel et par là même de leurs propres mouvements. Une telle science se traduit par un vocabulaire spécifique; et la maitrise de ce vocabulaire contribue au sérieux des connaissances. L'anatomie va donc également être un processus de désignation des divers os et muscles, les noms de ceuxci leur donnant existence et constituant parallèlement une classe de vocables.

« Je connais pour ainsi dire pas l'anatomie. Je sais pas donner des noms. L'anatomie, c'est donner des noms quand on veut en parler, oui. À un moment donné, il faut, sinon on peut pas la comprendre si on donne pas des noms à chaque... Moi, je me comprends, mais si j'en parle à quelqu'un, il va pas comprendre. Bon, un nageur, on lui montrera, mais quelqu'un de l'extérieur il va pas comprendre. » (Audrey, B. 2001)

Maîtriser la topographie du corps, c'est en maîtriser les lieux-dits; la cartographie se change en une nomenclature. La connaissance du corps humain est subsumée sous celle d'un lexique et la science anatomique s'accompagne de celle des noms.

La dénomination accompagne la description: connaître l'anatomie, c'est savoir attribuer des noms aux éléments osseux et musculaires dont il peut être question. Il ne s'agit pourtant plus d'un vocabulaire commun, trivial, mais d'une nomenclature savante qui contribue à la justesse de la connaissance.

«Ce qu'on a eu comme cours était purement nominatif... c'était descriptif. Le seul aspect fonctionnel pour moi était de savoir ce qu'un muscle faisait par rapport à un autre... c'était comme du trivial pursuit; voilà, on sait des choses qu'on peut replacer et montrer un peu qu'on est instruit mais je vois pas vraiment le rapport avec mes pratiques. » (Cyril, C. 2001)

53 Ces regrets formulés concernent l'inadéquation du savoir anatomique aux attentes des étudiants : comme science descriptive d'une réalité constituée, l'anatomie ne répond pas à leurs aspirations; elle n'est pas congruente avec les valeurs qu'ils lui attribuent, valeur d'utilité autant que d'épistémê.

«L'anatomie est nécessaire... connaître les structures du corps en soi n'a aucun sens. Il faudrait partir des structures et recontextualiser dans les APS. L'anatomie, en STAPS, devrait être enseignée de façon fonctionnelle; une structure anatomique doit être contextualisée. L'anatomie, c'est tout le désincarné par rapport à l'être. C'est l'étude de la pure biologie, mais qui réduit l'étude de l'humain à une seule dimension. » (Xavier, G. 2001)

Ce que souligne Xavier G., c'est la pauvreté pratique du savoir anatomique. La réduction au seul regard physique porté sur le corps "désincarne » ce dernier, alors même que la fonction primordiale assignée aux cours d'anatomie par les étudiants est de fournir une base rationnelle aux mouvements du corps humain, toujours conçus comme actes.

Si l'anatomie est " une science précise concernant des faits », elle se situe explicitement dans une ontologie dualiste et sépare la dimension physique de la "vie intérieure ". L'anatomie devient sous cet angle épistémologiquement superficielle. Or, le rôle qui lui 
est assigné par les étudiants reste central, relativement à leurs pratiques sportives. Il devient de ce fait nécessaire, pour eux, d'élaborer une anatomie adéquate qui réinsère la dimension descriptive dans l'expérience du corps propre.

Il s'agit dès lors d'abandonner les aspects normatifs du savoir anatomique fondé sur la seule dimension physique du corps humain, pour reconstruire une anatomie fonctionnelle, l'unité du moi-corps (Mach 1984).

«Pour moi, c'est un petit peu "sciences rigides" dans le sens où il y a pas vraiment possibilité d'avoir une interprétation personnelle des choses... C'est plus par rapport aux normes. Je le vois comme ça.» (Stéphanie, D. 2001)

Les étudiants sont en quête d'un savoir qui intègre des « interprétations personnelles ", et on constate un renversement de la nature de la connaissance recherchée : posée a priori comme savoir utile et nécessaire, prescriptif des actes, conçue dans l'absolu comme science rigoureuse et nominative, l'anatomie ne prend sens, in fine, que dans la subjectivité. Non pas dans une subjectivité singulière, une individualisation, mais dans un rapport phénoménal à l'expérience, elle-même largement partagée par le groupe.

La construction pratique du savoir anatomiqueL'anatomie descriptive : une théorie sans réalité

La cartographie du corps, sa nomenclature précise, bien que parées des valeurs de logique, de rigueur et de précision, apparaissent aux yeux des étudiants comme pures élaborations théoriques, alors que, par contraste c'est leur utilité pratique qui est attendue.
«L'anatomie est un cours qui m'a choquée dans le sens qu'il était trop dur... c'était trop à plat. C'est-à-dire qu'il y avait beaucoup de choses à apprendre et c'était vraiment à apprendre pour l'examen. Des choses qu'on retient, sur le schéma, mais on ne sait pas dire après sur le corps où est placé tel muscle. Sur le schéma, on peut le placer, mais on visualise pas après ... c'est un savoir qui sert vraiment pour avoir l'examen, après on n'arrive pas à s'en servir dans la vie de tous les jours. » (Audrey, B. 2001)

Rigueur et précision schématiques sont des qualités qui font obstacle au regard porté sur le corps. Les cours d'anatomie apparaissent déclaratifs et construits sur les bases d'un réalisme théorique. C'est sur le mode de l'image pré-construite que la réalité se fabrique et non à partir du regard sur les choses. Les schémas qui constituent les illustrations du cours sont des substituts d'une matérialité mais le passage de cette réalité-là à ses configurations est négligé. L'anatomie apparaît dès lors comme abstraite, théorique, et nécessite des efforts de ré-élaboration de l'entité concrète du corps.

«Peut-être, s'il y avait comme un squelette par morceaux, des muscles pareils, comme un puzzle en fait, qu'il y ait les insertions sur le squelette et que les étudiants remettent les muscles là où ils vont... » (Élisabeth, B. 2001)

Mais cette demande est ambivalente. Lorsqu'on questionne les étudiants sur l'intérêt que pourrait avoir la dissection pour la connaissance du corps humain, les réponses traduisent une large réticence. L'autopsie ${ }^{10}$ n'est acceptée par certains (et ils se font rares) que comme expérience particulière, non comme méthode de scrutation.

« Peut-être pour l'apprentissage de l'emplacement des muscles eux-mêmes, ça reste dans le cadre de l'anatomie descriptive... mais dans ce sens-là, j'en vois pas particulièrement l'intérêt. c'est quand même pas commun de pouvoir voir à l'intérieur... C'est intéressant dans le sens où c'est une expérience. Pas forcément une expérience qui concerne l'anatomie, mais c'est une expérience et une vision des choses. » (Cyril, C. 2001) 
61 Le réalisme concret reste sur un plan représentationnel, celui de l'analogue : ce qu'il s'agit de connaître n'est pas la matière physique du corps humain, mais le plan de celuici. Le corps concret auquel les étudiants font référence reste une figuration de l'étant anatomique. Le savoir anatomique doit porter sur des « maquettes».

«C'est vrai que des fois on se représente mal certains muscles; on les voit que sur un schéma. Je pense pas que je serais prête à faire des dissections, même pour aller mieux voir dedans, personnellement, non. Les schémas, le squelette, l'écorché avec tous ses muscles, ça suffit. » (Marie-Aude, L. 1991)

Si nous avons parlé de "réalisme concret » (différent du « réalisme schématique » que promeuvent les cours actuels), c'est pour souligner l'importance de la ré-élaboration représentationnelle du savoir anatomique chez les étudiants. Certes, ce dernier est abstractif tout en restant lié au concret. Mais la connaissance anatomique du corps est surtout connaissance d'une image du corps. Ce n'est pas la matière qui est concrète, c'est sa représentation, un mode analogue de figuration. Ainsi la planche 4, photographie d'une préparation anatomique d'une partie du thorax humain, suscite des jugements paradoxaux.

"C'est pas anatomique parce que c'est trop vrai, ça. C'est une photo, on dirait un cadavre ouvert... alors que pour moi l'anatomie, c'est du papier, c'est un os... c'est des schémas tandis que là c'est ouvert, on voit, là. Et c'est rouge!» (Corinne, $\mathrm{P}$. 1991)

La matérialité brute du corps n'est pas le concret auquel les étudiants font référence et, pour être acceptée, sa représentation nécessite une reconstruction représentationnelle.

Le déni de la réalité passe par sa simplification : elle est acceptable parce qu'elle a la nature d'une épure. Elle devient illustrative d'un schéma qui la concerne selon une opération mentale de renversement de la référence. De réalité, elle se change en image. Confrontés à un enseignement de plus en plus abstrait, les étudiants s'arrangent pour élaborer une anatomie faite de représentations d'une réalité factice, bien que concrète. L'exactitude des connaissances est minimisée, puisque celles-ci ne se rapportent pas au corps humain physique. De science exacte qu'elle était, l'anatomie se fait fantaisie.

La relativité du savoir anatomique

L'anatomie n'est pas atteinte dans sa spécificité ni dans ses caractères, mais elle est disqualifiée dans son statut de science d'appui pour les pratiques physiques.

«J'ai une cohérence approximative sur l'anatomie, mais... pour moi l'anatomie n'est pas une connaissance en elle-même, dans le sens où elle ne peut pas faire comprendre les choses. Elle peut à la limite participer à une explication, mais pas à la compréhension d'une chose... La compréhension d'un mouvement... si pour expliquer un mouvement, on peut passer par plusieurs points de vue, et l'anatomie en sera un. » (Cyril, C. 2001)

L'informateur remarque de lui-même l'incohérence entre une conception prescriptive du savoir scientifique qui lui donnerait sa valeur, et les limites épistémologiques qui se font jour. Si l'anatomie a un intérêt, c'est comme science explicative -effort de détermination causale du mouvement- et non comme fondement des pratiques. On relève là un heurt des représentations : la croyance en la positivité et en l'utilité du savoir anatomique s'affronte aux pratiques qui la contredisent. Vérité enseignée mais non justifiée, ce savoir perd ainsi son statut épistémique et devient croyance théorique sans cesse confrontée à une foi pragmatique inscrite dans les usages sportifs.

En effet, deux anatomies se côtoient : celle du corps propre, qui relève de l'expérience subjective, inscrite dans une réalité de l'acte, et celle, descriptive, d'un corps physique 
détaché de tout acte. La seconde devient anecdotique alors qu'elle constitue en partie le programme scientifique des cursus en STAPS. L'anatomie est un savoir inconsistant pour les étudiants, ce qui semble constituer un paradoxe au regard des pratiques qu'ils développent.

«Jusqu'à présent, j'en ai jamais fait, et puis j'entraînais bien des enfants et j'arrivais

à peu près à avoir des résultats quand même... » (Hervé, L. 1991)

68 La pratique sportive n'implique pas a priori une maitrise de la connaissance anatomique du corps, mais elle suppose l'élaboration de représentations de ce corps, représentations qui œuvrent comme véritables modèles du corps et des gestes.

Ce n'est que lorsque l'acte est accompli selon le modèle que sa compréhension devient nécessaire dans l'optique d'un progrès de la performance. La connaissance anatomique prend alors le visage d'un savoir "savant» qui complète et rectifie les fantaisies représentationnelles du corps. Mais c'est moins la précision du déterminisme physique $\mathrm{du}$ mouvement qui autorise un progrès de la performance, que l'effort de rationalisation a posteriori que le savoir anatomique permet d'opérer. La fonction de ce savoir est alors essentiellement psychologique. L'importance de la connaissance anatomique du corps devient très contingente, le corps physique s'effaçant dans les représentations des étudiants devant un corps phénoménal, composé complexe de sensations physiologiques et d'impressions psychiques.

«J'essaie plus de les faire réfléchir sur les sensations qu'ils ont. Les sensations... Au niveau corporel et au niveau psychique, comment ils ressentent la situation... Disons que c'est intéressant, mais si c'était plus une anatomie fonctionnelle, c'est-àdire comment on peut la rapporter par rapport aux sensations et par rapport à soi. »(Stéphanie, D. 2001)

Le savoir anatomique change de nature: la description rigoureuse de l'organisation physique du corps se transforme en composition de représentations et de croyances qui trouvent leur source dans l'expérience pratique.

Anatomie de l'expérience, expérience de l'anatomie

71 Le savoir anatomique prend sens dans l'expérience pratique. Ce sens est partagé, il est celui d'un groupe, celui des acteurs de l'activité vécue. L'intérêt porté à la connaissance physique du corps est relié à son usage. Ainsi la structure du corps se compose-t-elle en fonction de l'intervention des membres dans le mouvement et les gestes des sports, tels qu'ils sont développés par le discours technique.

«Dans le cyclisme, ce qui compte, c'est la position sur la selle et la tension sur le guidon. En tant que pratiquant ça [l'anatomie] m'a permis de sentir plus que ce que l'entraîneur me disait. L'anatomie est plus riche dans les techniques complexes.» (Xavier, G. 2001)

72 Il existe deux anatomies dans les représentations des étudiants : la connaissance vient se superposer au savoir d'expérience. Elle fournit non pas une élucidation, mais une illustration; elle montre plus qu'elle n'explique et principalement, elle permet de nommer ce qui jusqu'alors n'était qu'éprouvé, participant par là même au discours technique.

73 Le «corps technique " est celui d'une nomenclature partagée, un montage qui fait l'acte, serait-ce sur le mode de la fantaisie. Ce qui est retenu de cette nomenclature est issu de l'expérience personnelle. La connaissance perd tout caractère de positivité; elle n'est pas non plus circonscrite à un discours technique, elle est contaminée par les perceptions et sentiments de ceux qui la formulent. 
«Le tibia, je l'aime bien, le nom et l'os, et j'aime bien là où il est. Je trouve que la jambe c'est plus facile, c'est plus concret ... L'épaule, j'aime pas du tout; tout ce qui est là au milieu... encore l'épaule ça va, mais la cage thoracique, la colonne vertébrale, c'est trop compliqué, je trouve. Les jambes c'est important. Ça me plaît, je fais de la course, ça joue sûrement. » (Corinne, $P$, 1991)

Pour Corinne P. comme pour les autres étudiants, le corps est variable dans son absolu. Le savoir anatomique procède d'un jugement formulé à l'aune de l'expérience. Ce qui est primordial pour l'un est négligeable pour l'autre, et le jugement est fondé sur des éléments non cognitifs : ce n'est pas la structure anatomique du corps véritable qui permet le jugement, mais des opinions qui mêlent croyances constituées, sentiments esthétiques et foi pragmatique. C'est l'expérience éprouvée qui construit la connaissance du corps, lequel ne garde de positif que les expériences segmentées, parcellaires.

Il s'agit plus de saisie intuitive que de savoir contrôlé : une conscience de la topologie corporelle prend lieu et place de la description topographique du corps. Celui-ci est connu par ses lieux et non pas analysé dans ses structures, et ces lieux sont variables selon les expériences sensibles vécues par les étudiants.

Connaître est un acte empirique -et que peut-on mieux connaître que son corps ? Et pourtant, l'écart existe entre la sensation éprouvée pendant l'exercice et le repérage lucide, précis des muscles et des articulations en jeu. Le savoir anatomique est pour les étudiants la condition d'un contrôle possible; il n'éclaire pas mais vérifie et par là même, les dédouane de ce qui n'est que représentation. L'exercice offre la possibilité de telles élaborations anatomiques, mais un corps intègre est indifférent, malgré la sensibilité proprioceptive pourtant largement sollicitée lors de l'entraînement sportif. Blessures et douleur ordonnent la topographie imaginée du corps et le savoir anatomique est celui des lieux de lésions et de souffrance.

Une anatomie de la douleur

«L'anatomie, il faut la vivre. La douleur est un moyen de connaître son corps. Ça

permet de localiser. » (Cécile, E. 2001)

Prévenir la blessure, en mesurer la gravité éventuelle, est l'une des fonctions d'utilité reconnues à la connaissance anatomique. Mais cette fonction ne trouve une expression réelle chez les étudiants que lorsque la blessure survient. Celle-ci fait office de preuve empirique, et la curiosité est guidée par la force des impressions comme par la profondeur des regrets.

78 Atteindre la vérité du corps anatomique passe par l'épreuve. "Souffrir un peu pour l'anatomie ", c'est souffrir pour se saisir en conscience, c'est aussi souffrir pour accéder à un savoir. La douleur devient un acte épistémique en ce qu'elle permet de réaliser "d'une façon vraie» ce qui n'était que réalité théorique ou élaboration factice. La douleur est autopsie et recouvre le modèle habituel du connaître des étudiants; non pas mode expérimental, naturaliste, mais mode phénoménal.

79 Le siège de la souffrance est l'objet de tous les intérêts, de toutes les interrogations. Mais la curiosité n'est pas neutre : elle repose initialement sur un fond d'inquiétude. Le savoir anatomique $\mathrm{a}$, là encore, une fonction principalement psychologique. La douleur irradiante se mue en curiosité focalisée, en véritable épistémophilie. Le segment blessé devient l'endroit préféré du corps, celui sur lequel portent toutes les attentions, celui pour lequel la conscience est acérée par le savoir. 
« Moi j'avais fait une fracture du pisiforme, j'avais demandé ce que c'était parce que j'en savais rien du tout... Et donc, bon, j'ai demandé exactement ce que c'était et après j'ai sorti une planche pour voir quand même, pour essayer de me faire une représentation de l'image parce que... même à la radio, si, à la radio, je voyais un petit truc mais... c'est pas précis précis, quoi... j'aime pas avoir des doutes sur des blessures ou des choses comme ça au niveau de mon physique... Déjà j'ai pas tellement confiance dans le milieu médical en général alors, je suis curieuse et pénible parce que j'aime bien comprendre. » (Nathalie, F. 1991)

Là encore, le savoir anatomique a un rôle de vérification. Tout comme pour le geste sportif, c'est un souci de repérage, de contrôle a posteriori qui est à l'œuvre, et la topographie du corps devient carte des blessures ou blason des douleurs éprouvées, musculaires, osseuses ou articulaires. Dès lors, la composition corporelle varie et ce sont tantôt les os, tantôt les muscles qui constituent la chair du corps.

«Moi, j'ai eu une fracture des cervicales, en fait je me suis rendu compte de la gravité de la blessure qu'après... quand on m'a montré les radios, qu'on m'a montré, bon, que c'était plus ou moins important, ce qui aurait pu se passer... Pour moi la colonne vertébrale, c'est la partie du corps la plus importante... Il y a bien les insertions, le trajet des muscles, quoi... ce qu'on appelle l'insertion des muscles, son trajet, son action... Mais moi, comme je me suis fait plus de fractures que d'entorses, luxations... c'est vrai que je me pose moins la question. » (Nathalie F. 1991)

81 Pour une étudiante, qui fait état de plusieurs fractures subies durant les activités physiques, la connaissance anatomique est avant tout celle du squelette, structure solide susceptible de se briser. Telle n'est pas l'anatomie de la blessure musculaire, qui est rarement rupture : c'est une désorganisation douloureuse du geste qui est ressentie, et l'exploration du corps est une traque, la connaissance du siège de la douleur une véritable découverte.

"J'ai été blessé. On n'a pas trouvé sur le coup mais c'est au niveau du... pectinée, donc c'est à l'aine... je me rappelais pas de ce muscle... et j'ai été voir plusieurs médecins, et au départ on n'a rien trouvé; on a fait des échographies et des radios et on ne trouvait rien, et en fait, une fois, j'ai été voir monsieur D., il a une méthode assez particulière pour soigner et on a trouvé ce petit muscle... On a trouvé ce muscle parce qu'il était profond. » (Fabien, C. 2001)

82 Ce qui est mis en évidence par ce témoignage, c'est combien la recherche et la connaissance sont partagées: Fabien C. est autant que le médecin l'inventeur de sa blessure, le découvreur de son anatomie, que confirme le nom du muscle lésé.

Le savoir anatomique, chez les étudiants, est paradoxalement connaissance incarnée. C'est en référence aux expériences vécues qu'il s'élabore et son vocabulaire opère comme confirmation. Savoir, c'est nommer ce que l'on connaît déjà et qui est le corps propre. Le squelette, l'écorché, le schéma permettent la reconnaissance. Essentiellement phénoménale, l'anatomie éprouvée trouve dans l'anatomie sue un mode de confirmation qui ne peut guère servir à modifier ni même à comprendre les pratiques sportives. Pour les étudiants, l'anatomie n'est pas un savoir naturaliste descriptif d'une réalité matérielle constituée; elle est une fabrique active de corps, fantaisiste et impressionniste, et toujours elle exige un savoir-vivre.

Le savoir-vivre anatomique

De la façon dont les étudiants l'envisagent, l'épistémê anatomique est une science de la vie - mais elle enferme dans une aporie.

«Oui, mais l'anatomie, c'est mort! C'est mort tout en étant vivant, je veux dire que... c'est difficile ! C'est un squelette, là, mais il est en mouvement et le squelette quand on l'étudie... c'est pas en mouvement. » (Corinne, P. 1991) 
(ammentaire, exprimé à propos de la planche 7, trahit l'impasse logique dan laquelle se placent les étudiants. L'anatomie, comme science de la structure matérielle $\mathrm{du}$ corps, donc exploration naturaliste du cadavre, se confronte à l'anatomie impressionniste et phénoménale de l'expérience pratique révélée dans les activités physiques. Le corps qui est là est double, avec son anatomie du dehors et celle du dedans, reliées l'une à l'autre par l'enchantement d'une connaissance.

Le dedans et le dehors

La peau est une limite, une frontière. Toujours posée comme enveloppe corporelle, elle est le plan où l'anatomie commence. Plan ambigu pourtant, puisqu'il pose le problème insoluble du recouvrement de l'anatomie phénoménale par l'anatomie physique. La peau n'est pas rattachée à l'anatomie, elle relève d'un autre ordre de connaissances.

«La peau n'a rien à voir avec l'anatomie? Non, non. Parce que l'anatomie, c'est avant tout l'étude des muscles et des os; l'action des muscles sur d'autres. Alors la peau, on n'en parle pas. Dans un exercice, par exemple de musculation, il faut solliciter les muscles, la position des membres... on parle pas de peau. Quand on fait des mouvements, c'est autre chose; c'est différent, on sue. C'est pas de l'anatomie, je suis persuadée que non. La peau revêt le corps humain, c'est une couverture. » (Nadia, L. 1991)

"L'anatomie ce serait plutôt ce qui est interne... ce qui met en mouvement. La peau c'est l'enveloppe. Les enfants en gym, je vois l'ensemble, pas un muscle, un os. Le squelette est peut-être une représentation qu'on se fait et la peau est ce qu'on perçoit réellement. Je pense que la peau a à voir avec la physiologie. » (Marie-Aude, L. 1991)

L'enveloppe corporelle doit être franchie pour que le territoire de l'anatomie s'offre à la découverte. Le passage de la personne à l'anatomie s'opère par une écorchure: le retrait de la peau offre l'entrée dans le territoire des os et des muscles. L'exploration, pourtant, malgré sa qualité de facticité, ne saurait être une aventure. S'installe ici une stratification du possible et du permis.

La géométrie corporelle est altérée dans sa perspective. L'anatomie débute sous la peau mais reste dans une périphérie dont le centre est le squelette osseux. L'« intérieur » est apprécié selon les mêmes critères que ceux appliqués à la peau. Ramenée aux muscles et à la charpente osseuse, l'anatomie perd sa nature organique mais aussi toute réalité tissulaire.

« En plus il y a des moulages là qui se font... on a un beau muscle tout moulé... c'est esthétique à la rigueur... c'est pour ça je reste là-dessus quoi, je me représente, je vois rien, je vois rien au milieu, je vois un muscle, l'os, l'insertion et c'est vrai que je vois pas le reste c'est-à-dire le tissu, le liquide dans lequel il est, etc... c'est vraiment très propre. » (Nathalie, F. 1991)

Les témoignages confirment la facticité de la réalité concrète concernée par le savoir anatomique des étudiants. Le corps organique, naturel, objet apparent de ce savoir, ne peut être envisagé, ni même vu. Seul peut exister un corps de représentation, c'est-àdire une réalité imaginée mais qui est susceptible de se montrer. Réalité de pure forme, le corps du savoir anatomique des étudiants est un corps sans organe ni tissu.

Un corps sans organes

"C'est vrai que pour moi le cœur est plus un organe qu'un muscle. C'est vrai que pour moi, tout ce qui est musculaire, c'est vraiment tout ce qui est visible. Je crois que c'est ça... Tout ce qui est... les bras, les jambes, tout ce qui n'est pas interne. » (Stéphanie, D. 2001)

Techniques \& Culture, 39 | 2002 
90 lecture est rattachée à l'autorisation du regard. Est visible ce qui, pour l'informatrice, est vu, c'est-à-dire l'action des membres et le dessin des muscles sous la peau. La vision est morphologique dans sa constitution première. Elle est aussi anatomique, car la surface du corps n'est que la périphérie de son milieu, non de son intérieur qui est le territoire des organes. Ce qui est visible, c'est la morphologie, ce dont la représentation est autorisée : aux yeux des étudiants, le corps anatomique est un entre-deux de la peau et des organes. Il est réduit à ce qui, déjà, est visible dans le geste sportif, ou du moins, à ce qui y est deviné ou perçu, donc autorisé et nécessaire comme objet d'un savoir.

ne contradiction fondamentale, déjà soulignée, est ici redoublée -entre la connaissance de l'anatomie physique du corps humain et les agencements représentationnels liés aux actes. L'anatomie du cadavre n'est pas la vie, mais reste la référence matérielle de la possibilité du mouvement. La vie naturalisée réside dans les « organes » qui sont exclus de l'anatomie; la représentation des étudiants est, dans ce cas, vitaliste et biologique. Existe dès lors une profonde antinomie entre l'exigence de rattacher l'anatomie à la vie et le refus de se soumettre à l'anatomisation des «organes ». Le corps que se représentent les étudiants est un androïde anatomique; il reste profondément rattaché à une métaphysique mécaniste.

«Il faudrait que tout le monde puisse le voir en action, mais... ça doit coûter très cher, un squelette de ce type, où on puisse voir comment le muscle se contracte, comment le muscle antagoniste subit un étirement... voir vraiment comment marche le squelette humain... Faudrait voir si le squelette peut bouger, lui faire appliquer un mouvement. Après, c'est chacun dans son sport de visualiser... » (Audrey, B. 2001)

Modèle factice du corps, l'androïde anatomique est un modèle analogique du mouvement. Il prend valeur vitale parce qu'il est puissance de mouvement, mais cette valeur est une illusion contrôlée puisque non reliée aux organes vitaux. À partir de là, la différenciation sexuelle des corps n'a plus d'importance; elle est réduite à des détails relatifs à la forme du squelette ou au volume et à la masse des muscles.

«Chez l'homme ou la femme, c'est bien la même anatomie, à peu près... au point de vue musculaire, squelettique et osseux... ça varie pas beaucoup.» (Rodolphe, B. 1991)

" Anatomiquement parlant je crois pas qu'y ait de différences extraordinaires entre [l'homme et la femme]... on les cite les différences, oui, on les cite, notamment au niveau du bassin ... mais... ça voudrait dire que l'étude porterait uniquement sur un sexe donné. » (Pascal, B. 1991)

La différence des sexes ne se place pas dans l'anatomie. L'andrö̈de anatomique n'a pas de sexe, ou s'il en dispose, il sera homme.

«Le squelette... c'est plutôt un homme, je sais pas pourquoi. Parce que toutes les expériences qu'on fait, c'est toujours l'homme qui est sollicité. Pourquoi ? Et la femme non, elle est toujours... Peut-être que l'homme est plus fort, mieux construit, mieux bâti. Un squelette de femme, c'est... moins montrable et puis tant mieux pour nous. On n'est pas des exhibitionnistes. On est toujours resté sur l'image de l'homme en tant qu'être et en tant que modèle. La femme, c'est autre chose. » (Nadia, L. 1991) ${ }^{11}$

La partition sexuelle ne saurait s'appliquer au modèle du corps anatomique chez les étudiants. La présence d'une figuration du sexe sur les planches anatomiques peut même constituer un obstacle à leur reconnaissance. Montrer le sexe, c'est montrer la 
vie, c'est appréhender le corps autrement que selon le réalisme concret d'un androïde anatomique -ce dont témoigne le commentaire que fait Corinne P. de la planche 1.

«Oui c'est anatomique, mais toujours pareil, il y a le sexe... Il y a pas la peau, on voit mieux. Le sexe, on l'étudie pas en anatomie, c'est à part. Ça ne fait pas partie de l'anatomie, on n'en parle pas, je pense pas. Moi, ça me plaît parce qu'on voit bien les muscles... justement c'est bien dessiné... c'est un ensemble... on voit tout. Il paraît vivant... parce qu'il a une main tendue, parce qu'il y a son sexe, parce qu'il a un pied levé, parce qu'on voit ses oreilles. » (Corinne, P. 1991)

La représentation du corps trouve sa limite dans les indices de la vie. Pour qu'une figure se révèle anatomique, il faut que l'organique en soit absent ou minoré, que la vie soit circonscrite aux mouvements possibles mais qui jamais, dans la froideur d'un savoir, ne sont autre chose que les courses réglées des segments d'un mécanisme. Ce corps est un corps sans organes. L'autopsie est donc interdite, impossible, car elle dévoilerait ce qui justement n'est pas à voir. Ouvrir un cadavre, y entrer pour voir, ne peut être qu'une expérience psychologique, quelque chose de douloureux à vivre.

«La dissection, ça aurait un intérêt... un intérêt à sa juste valeur : ça permet le rapport corporel entre le vivant et le non-vivant. » (Xavier, G. 2001)

La qualité d'une telle expérience ne serait pas cognitive, ne répondrait pas à un désir de savoir : c'est sur le plan métaphysique qu'elle prendrait sens dans la confrontation de l'être à sa dépouille. Pour les étudiants, elle apparaît davantage comme épreuve métaphysique, ontologique, que comme possibilité de connaître.

Les représentations de l'anatomie humaine, nous l'avons dit, mêlent étroitement chez les étudiants le corps-sujet et le corps-objet, le corps phénoménal et le corps matériel. Mais jamais ce dernier n'occupe la référence en tant que réalité. L'entité concrète dont il est question n'a de réalité que mentale ou pratique -et non physique.

En cela, l'anatomie du corps est reliée à l'expérience; elle est pragmatique mais garde un rapport à la seule matérialité du corps; elle « représente plus un outil que quelque chose d'existentiel ». Elle se pose comme intermédiaire concret entre le théorique et le matériel, mais aussi entre l'étant et l'existence. Pour les étudiants, la représentation de l'anatomie du corps humain ne se résume pas à un montage phénoménal, à une fantaisie perceptive qui adhère à l'expérience; elle témoigne aussi du passage incessant du vivant au mort, de l'étant à l'existence.

Le mort et la vie

Le cadavre est ambigu : en lui subsiste le souvenir de ce qu'il n'est plus. Il constitue un rappel, un regret de la vie. Il est stigmate plus que matière.

« On peut mettre en relation le corps, ce qu'il en reste, l'aspect morphologique et physique de ce corps avec l'ensemble des pratiques susceptibles d'avoir influencé ce corps, la taille des muscles... » (Cyril, C. 2001)

100 Toujours, resurgit l'anatomie pragmatique où le cadavre n'a pas sa place. Il s'agit derechef d'un faux, issu de l'esprit, d'un effort d'imagination, nécessairement représenté et non pas regardé.

"Qu'est-ce que vous voulez qu'on fasse avec un vrai squelette, non... Vaut mieux que ce soit un faux, ce serait quand même du gâchis. Un vrai, ils peuvent faire ce qu'ils veulent avec, ils peuvent étudier les os; en médecine, ils en ont plus besoin que nous; pour eux c'est important. Pour nous, ce qui nous intéresse c'est la forme, c'est tout... » (Corinne, P. 1991)

Le cadavre est une impossibilité, la matière une abstraction. La réalité n'est pas constatée, elle n'est pas regardée, elle est de l'ordre du songe. L'anatomie du corps 
humain est nécessairement illusoire. La description anatomique ne répond pas aux soucis pratiques des étudiants et par là elle n'assume pas le rôle d'une connaissance. Le mort est un obstacle au songe et suspend le désir de savoir, de saisir la réalité.

«L'anatomie n'est pas vivante mais réelle... je crois. Moi, personnellement, j'apprécierais pas de voir la réalité, enfin je pense... Je préfère la voir sur des livres... pour moi, une étude sur un être humain réel, ça me choquerait peut-être, ça me retournerait peut-être un peu... » (Marie-Aude, L. 1991)

Les représentations balancent entre la vie et la mort qui toujours fait retour comme réalité funeste. L'anatomie a donc un rapport inéluctable avec le cadavre qui, par le jeu des images, est cadavre en mouvement, dépouille animée. Le corps matériel est sublimé en structure animée qui, par son mouvement, fait semblant de vie.

«Ça a à voir avec une description, pas avec la vie. Je dirais pas forcément avec la mort, mais un peu, puisqu'elle a basé sa connaissance à partir de la mort, puisque c'est en étudiant les morts que l'anatomie s'est créée. C'est pas parce qu'elle a un rapport avec la mort qu'on peut pas s'en servir.» (Cyril, C. 2001)

Ce qui pose problème aux étudiants, ce qui alimente leur métaphysique complexe où se mêlent vitalisme spontané et mécanisme construit, c'est la force irréductible de leur expérience pratique que l'anatomie les contraint à confronter avec le fatum de la mort. En recomposant le mort en androïde anatomique, ils revitalisent la mort.

Pour les étudiants, l'anatomie naturaliste, descriptive de la structure matérielle du corps humain, ne peut représenter, malgré ses caractères de positivité et de rigueur, le noyau d'une connaissance. Constamment ramenés à leur expérience pratique, qui reste à leur yeux la source première de leurs connaissances, ils réélaborent le corps humain dans leurs représentations et croyances et font de l'anatomie un effort de vie, une pulsion de savoir-vivre.

Commentaires des planches anatomiquesPlanche 1

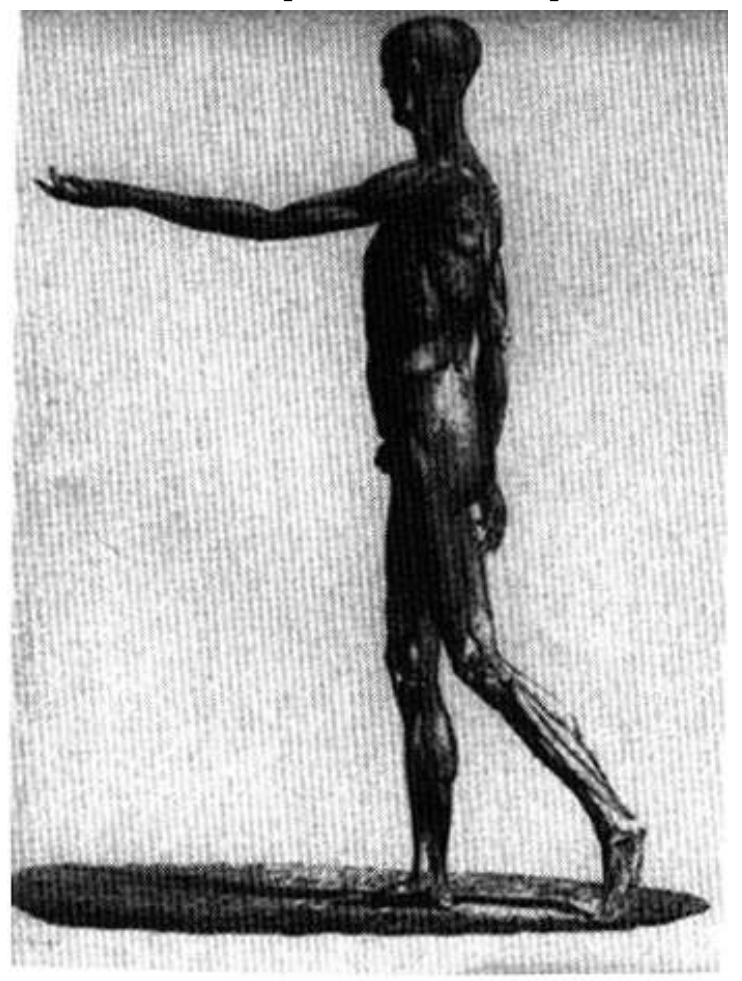

Planche 1. Icônes anatomicae. Léopoldo Marcantonio et Floriano Caldani, 1801 
«Elle permet de voir les muscles superficiels. Oui... elle permet de mieux se représenter les muscles... ici, on voit beaucoup mieux. Ça aide au niveau des représentations. » (Marie-Aude, L. 1991)

La planche 1 suscite peu de commentaires, et ceux-ci se formulent essentiellement en termes de précision du dessin, de piqué du trait. Elle permet des représentations, des impressions de ce que l'anatomie humaine peut être. Le corps va de soi et cela dans le double sens de l'évidence, qui ne peut produire qu'un jugement esthétique, et d'un corps en marche, qui n'entraîne guère de contradictions avec la vie.

«Moi, ça me plaît parce qu'on voit bien les muscles... justement, c'est bien dessiné... c'est un ensemble... on voit tout. Il paraît vivant... parce qu'il a une main tendue, parce qu'il a un pied levé, parce qu'on voit les oreilles. » (Corinne, P. 1991)

Planche 2

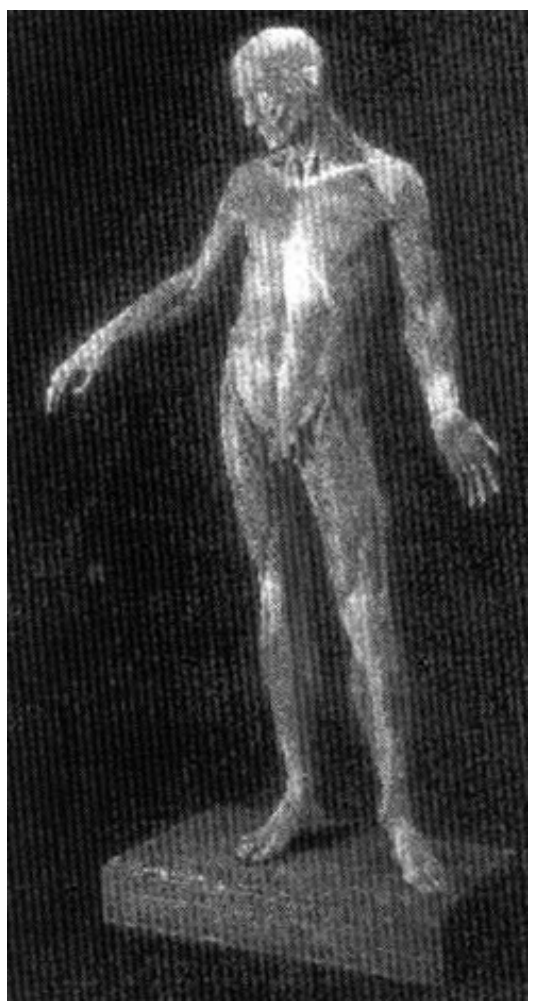

Planche 2. Modèle en cire de Pinson (XVIIle siècle), Cabinet d'anatomie du Duc d'Orléans

Cette planche relie sentiment esthétique et représentation de l'anatomie; elle constitue ainsi une référence représentationnelle, car en elle sont synthétisées toutes les contradictions que les entretiens révèlent.

«Ça, c'est plus esthétique... peut-être que c'est parce que je fais du dessin, c'est plus, enfin... plus artistique. C'est peut-être aussi au niveau couleur, c'est quelque chose qui m'a frappée. » (Marie-Aude, L. 1991)

«Là, ça représente vraiment... il n'y a plus la peau donc... là, on est plus dans l'écorché; on voit les détails musculaires, on voit les muscles. » (Fabien, C. 2001)

La réalité est reconstruite dans une facticité qui n'exclut pas le mort: le cadavre devient plastique et exposable. Le réel ainsi construit est anatomique parce qu'il fournit une image du corps osseux et musculaire, image du vrai parce que copie de la nature matérielle. Copie de fantaisie pourtant : l'écorché de la planche 2 joue avec la nature; il 
n'est matière qu'apparemment. S'il est une figure du mort, il l'est sur le mode du pentimento, du regret de « ce qui a vécu ».

«J'aime bien les couleurs. On a l'impression qu'il est posé là et qu'il se demande comment ça se fait. Donc, il demande qu'on lui réponde, pourquoi il est posé là... avec les mains... Peut être qu'il est trop vivant, c'est pour ça qu'il est pas assez anatomique. » (Élisabeth, B. 2001)

Ce commentaire souligne le paradoxe majeur auquel les étudiants sont soumis: l'anatomie vraie est une science du cadavre dont il est pour eux impossible de s'approcher, mais qu'il est toujours nécessaire d'accepter.

Planche 3

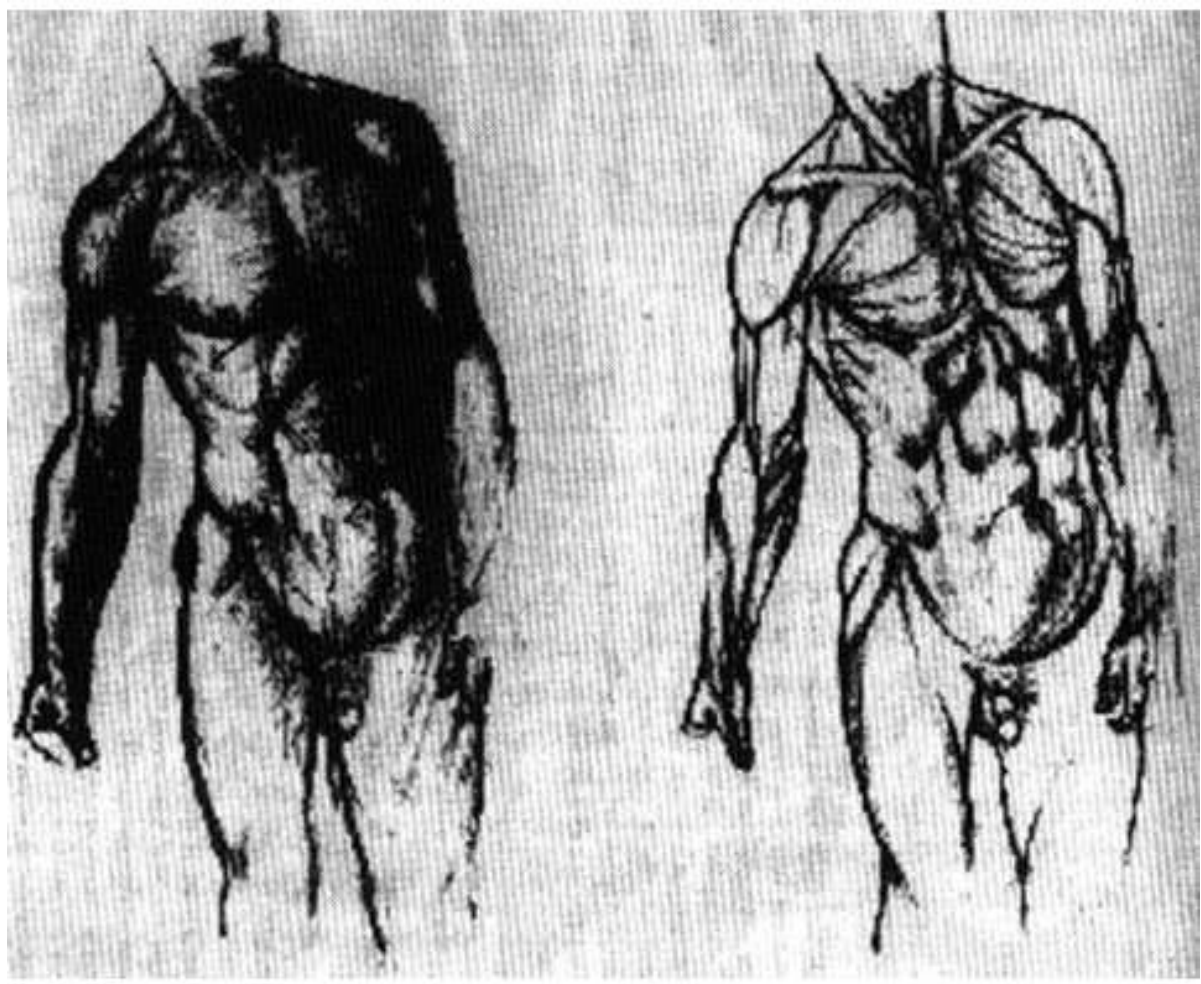

Planche 3. Anatomie artistique. Jeno BARCSAY (Berger-Levrault, 1982)

De façon surprenante, la planche 3 n'occasionne que des jugements esthétiques : si elle est appréciée, c'est pour la beauté d'un tronc musculairement harmonieux, alors qu'elle est le modèle de cette anatomie « qui lit l'intérieur de l'extérieur ». (Stéphanie, D.)

« Il fait plus dessin... c'est moins vrai. » (Élisabeth, B. 2001)

«Ça n'a aucun intérêt anatomique. » (Xavier, G. 2001)

«C'est un dessin, mais son appui pour le dessin, c'est l'anatomie.» (Marie-Aude, L. 1991)

110 La proximité d'une réalité en vient à gêner -réalité quotidienne et non anatomique. Si la planche 3 ne recueille que peu d'assentiments, c'est parce qu'elle est image d'une anatomie prosaïque, et non de l'anatomie épurée, sublimée à laquelle les étudiants en appellent.

Planche 4 

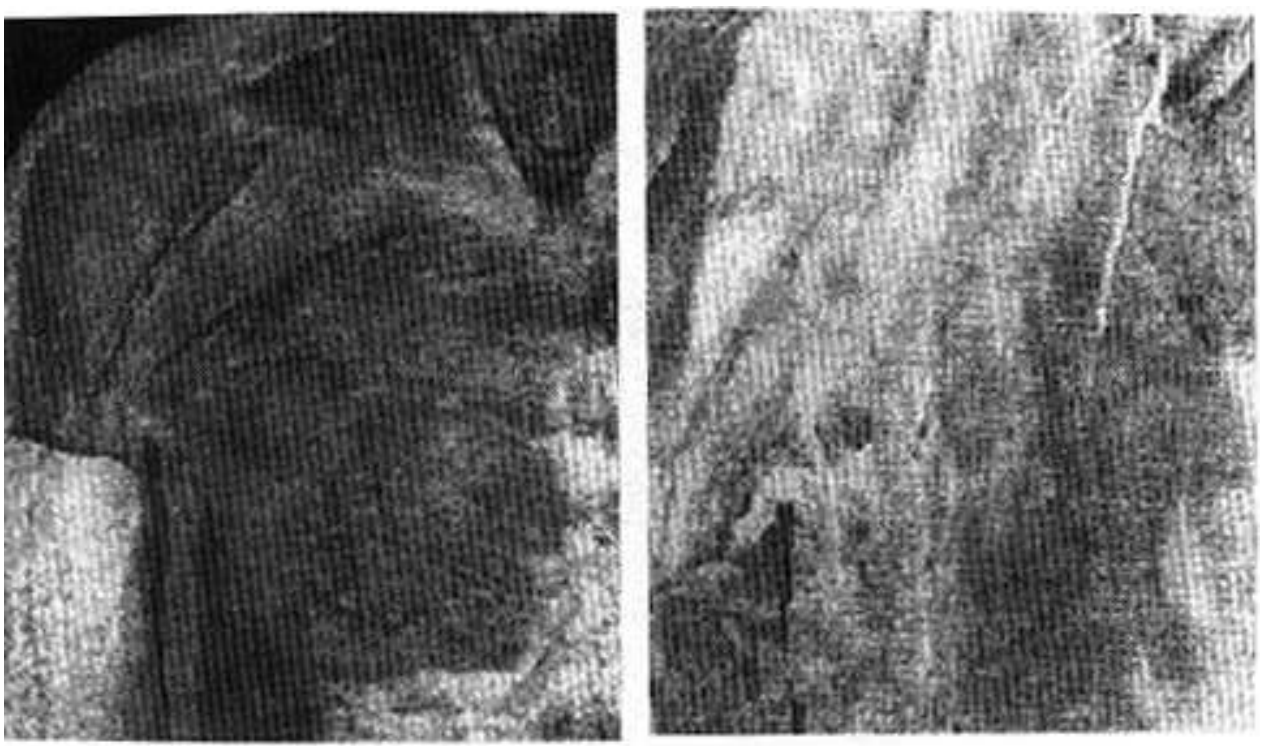

Planche 4. Atlas en couleur d'anatomie humaine (Édition Medsi, 1986)

111 La planche 4 suscite des commentaires ambivalents. Elle apparaît à la fois proprement anatomique et rejetée en raison sa matérialité même. La photographie présente les caractères majeurs attribués à l'anatomie - précision et clarté- et correspond à la façon de partager le corps en lieux anatomiques.

« On voit les muscles, ça a un intérêt pour nous. On voit des morceaux, des surfaces musculaires. » (Élodie, J. 2001)

112 Il s'agit non seulement d'une anatomie du lieu, mais également de l'entre-deux, de ce qui se situe sous la peau, et n'est pas encore la profondeur de l'intérieur.

«On voit... c'est une coupe... donc, il y a pas la peau; on voit les muscles tels qu'ils sont et leur insertion... on voit vraiment les muscles. Il y a plus rien qui les cache maintenant. » (Audrey, B. 2001)

113 Le mort est là, mais ne fait toutefois pas irruption de manière trop violente.

"C'est régional, on voit bien les fibres, leur orientation... celle-là est décontextualisée donc elle ne me fait aucun effet. » (Cyril, C. 2001)

114 Le rétrécissement de la perspective donne à la matière corporelle une image d'irréalité. La planche 4 est anatomique parce qu'elle est congruente avec les schémas étudiés; elle convient au réalisme théorique des étudiants. Elle est alors illustrative des représentations construites. Elle est anatomique aussi parce que la matérialité est là : en deçà du regard qui sublime, la matière physique s'impose et altère le jugement même. La matière corporelle est crue, et cruelle.

«Moi, je dirais, c'est pas anatomique parce que c'est trop vrai, ça. C'est une photo, on dirait un cadavre ouvert... alors que pour moi, l'anatomie, c'est du papier, c'est un os... c'est des schémas, tandis que là c'est ouvert, on voit là. Et c'est rouge !» (Corinne, P. 1991)

«Enfin, c'est pas de l'anatomie que nous on pratique... c'est vraiment recherché, plus approfondi, c'est le cas de le dire. Ça fait moins cru sur un dessin... qu'en photo. » (Hervé, L. 1991)

115 Advient ainsi un déni : la réalité substantielle elle-même ne peut être pour les étudiants qu'un fatum anatomique. Ce n'est pas d'elle dont il s'agit dans leur réalisme concret, même si son absence ne peut être affirmée. C'est donc inéluctablement qu'elle 
s'affirme, en deçà des élaborations concrètes de l'anatomie des représentations qui opposent beauté et fantaisie à la laideur prosaïque de l'intérieur du corps.

« Elle est pas belle! Je trouve ça laid. Ces os, ces muscles, j'arrive pas... Ce squelette, ce mort, cette morphologie... Des fois on a du mal à se dire "on ressemble à ça, à l'intérieur". C'est dingue! C'est dur. On se dit "on est comme ça, c'est bizarre". Je trouve ça lugubre; on a l'impression de se trouver dans le monde des morts.» (Nadia, L. 1991)

116 La substance désenchante l'anatomie, elle contraint au passage des représentations à la matière, et ancre la mort dans le mort.

Planche 5

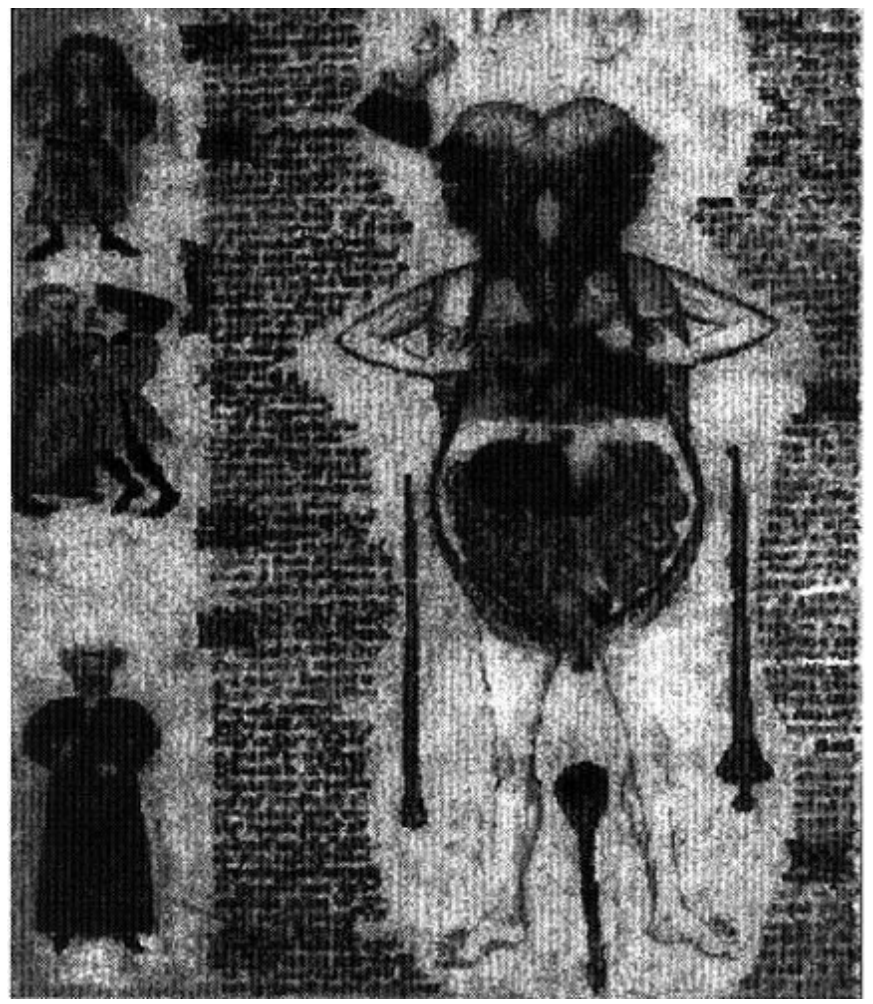

Planche 5.Dissection de Arte pluriali et chirurgica (John Arderne, 1412)

117 La conformation de l'image, qui confronte texte et peinture, prend une valeur autre que celle de la naturalité. La planche apparaît comme incompréhensible, dépassée, objet de jugements incertains. Elle n'est pas représentation du corps, mais bien figure, témoignage daté.

«Une page d'un livre religieux... je vois mal la conception de l'anatomie.» (Hervé, L. 1991)

«Ça fait un peu sourire, mais disons, c'est l'anatomie de l'époque, mais maintenant ce ne serait plus considéré comme de l'anatomie. » (Stéphanie, D. 2001)

«C'est les débuts de l'anatomie, mais non, c'est pas l'anatomie puisque ça, c'est les intestins, le foie, etc... C'est de l'anatomie mais pas la vraie. » (Corinne, P. 1991)

L'anatomique n'est relié qu'à l'ouverture du corps, un acte qui vainc une sacralisation, et non pas au regard lui-même, jugé confus et peu rigoureux, impuissant à déterminer un ordonnancement.

Planche 6 


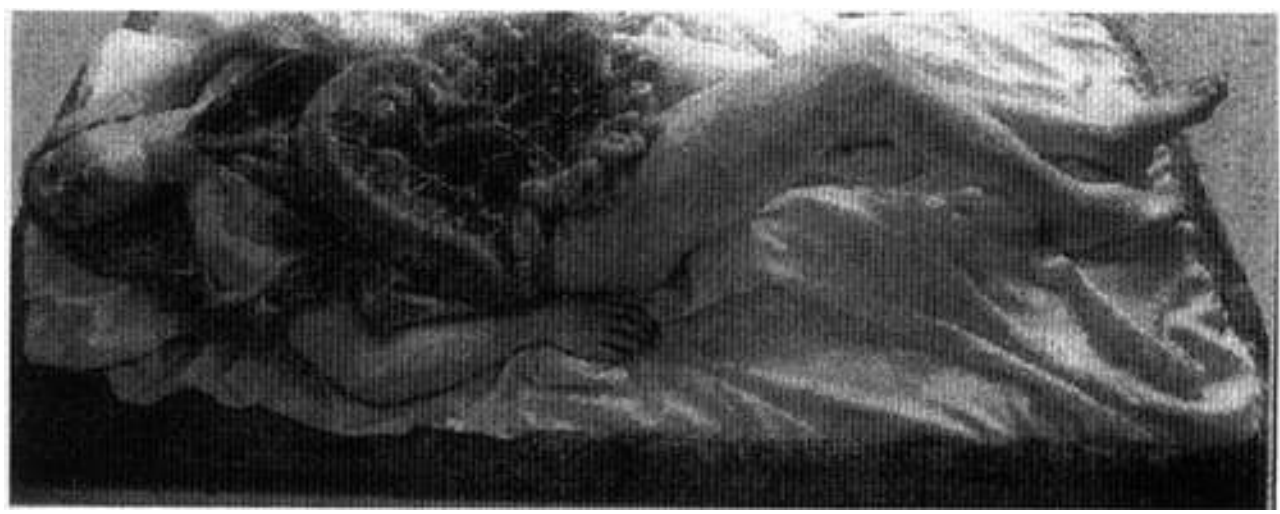

Planche 6. Vénus médicéenne (cire)

119 La vision de cette cire de la Vénus médicéenne provoque un flot de jugements qui tous, procèdent du rejet et parfois du dégoût. La planche 6 condamne à l'émotion et au paradoxe représentationnel. Il s'agit d'un corps, ouvert, de l'intérieur d'un corps, de sa profondeur, d'un corps de femme, mutilé mais entier malgré tout, d'une mort dans le vivant, d'un scandale de la réalité lorsqu'elle est substance; d'une blessure de l'entendement.

"C'est plus que de l'anatomie. C'est de l'anatomie pure. C'est le corps mis à

l'extérieur. » (Cyril, C. 2001)

120 Si l'image est acceptée comme anatomique, c'est avec réticence. Lorsque Cyril C. la qualifie d' "anatomie pure ", c'est à la substantialité du corps qu'il fait référence. Si l'anatomie est l'investigation de cette substantialité, elle n'est pas que simple constatation. Le regard anatomique suppose un détachement, une transcendance par rapport à la matière. Ce qui est vu n'est pas scrutable; il ne s'agit pas de connaissance, mais de spectacle. Or, l'anatomie garde fondamentalement la qualité d'un savoir et non uniquement d'un voir.

« On voit à l'intérieur, c'est... les organes vitaux mais ça apporte, je dirais, pas grand chose, mais... c'est quand même ce qui est à l'intérieur de notre corps et qu'on ne voit pas de l'extérieur. » (Audrey, B. 2001)

121 L'étalement des organes apparaît comme obscène et conduit à des impressions contradictoires. Les organes sont en quelque sorte l'intimité même du corps, ils sont " anatomie pure» parce que substance même de la vie, de sa profondeur; et les extérioriser, les rendre visibles, c'est supprimer la vie en en faisant voir la teneur matérielle. La vie n'est maintenue dans le mort que par anatomisation de celui-ci -non par sa présentation ni par son autopsie. La parcellisation de la cartographie anatomique, le découpage du corps en lieux, sa recomposition ou représentation permettent de créer une réalité factice qui n'est pas "pure anatomie », mais qui relie le savoir sur le corps à un savoir du corps placé dans l'expérience pratique. Aussi la planche 6 pose-t-elle problème, car elle provoque des tergiversations du regard porté sur l'anatomie du corps humain en gênant le détachement vers une transcendance.

«En fait, les boyaux qui ressortent, c'est pas quelque chose de très très joli; en fait, ils ont fait ressortir d'une femme qui est plutôt jolie... ça fait contraste. Comme elle est positionnée, on dirait qu'elle dort juste, avec la jambe relevée. En fait... elle a les boyaux... Je pense pas que c'est une dissection... je pense que c'est plutôt... un montage. » (Élisabeth, B. 2001) 
Cette informatrice -comme d'autres- fait l'hypothèse d'un montage et expose ainsi une manière de sublimer l'image qu'elle voit en séparant l'extérieur, public, «joli ", visible, et l'intérieur du corps que seul un événement transgressif de la vie peut exposer à la vue.

«Ben de voir comme ça l'intérieur, quoi, je suis pas trop habituée... c'est pas très joli d'apparence... c'est le côté mutilation, je crois, pour laisser voir ce qu'il y a dedans. Oui, je trouve que ça fait mutilé. » (Nathalie, F. 1991)

"C'est la plus anatomique, parce que c'est l'intérieur du corps... j'aime pas parce qu'on voit la personne, c'est-à-dire qu'on peut encore voir comment elle était avant... on peut voir sa réalité... c'est pas vraiment que j'aime pas, mais ça me fait un peu peur parce que tout le monde peut être à sa place. Il y a trop de vie dans ce cadavre. » (Stéphanie, D. 2001)

Le corps ne peut, dans les représentations des étudiants, être mutilé. L'écorché n'apparaît pas comme tel parce qu'il est une fiction et que celle-ci peut être parée des vertus de la beauté. On retrouve là l'entre-deux anatomique déjà évoqué qui constitue le concret de référence des étudiants. Il s'agit d'un véritable éloge de la beauté nécessaire à l'anatomie. Dans les représentations étudiantes, le corps anatomique procède d'une esthétique qui mêle la vie et le mort. Le mort est une abstraction anatomique pour les étudiants; il appartient au cadavre "qui a vécu » et qui est une élaboration représentationnelle. À l'opposé, la mort est une réalité émotionnelle, scandaleuse, qui n'est pas imaginable en réalité. L'anatomie du mort, du cadavre, n'exclut pas la référence à la vie parce qu'elle n'est qu'une anatomie de structure. Le spectacle de la mutilation au contraire annihile toute vie et centre le regard sur la mort, qui n'est pas réalité, mais question. Cette forme de sublimation est heurtée par la violence de la planche 6 , qui mêle, en une image, la beauté socialisée du corps et sa transgression par la mort. Elle est pour les étudiants un véritable scandale anatomique qui les prive d'une réalité concrète acceptable pour les précipiter dans la méditation métaphysique.

«C'est un film d'horreur comme c'est fait, l'ensemble. Ça fait penser à la mort. C'est une position pas naturelle pour la mort. Ça fait boucherie, accident de voiture.» (Cyril, R. 2001)

«On n'a pas de rapport à ça en STAPS... elle est trop vivante, trop vraie. Il ne faut pas que ça choque non plus... ça sert à rien. On a envie d'apprendre des choses en anatomie, mais d'apprendre l'anatomie... pas de déballer. » (Élodie, J. 2001)

L'impossibilité de regarder la planche 6 découle d'une difficulté de réification. Cette image ne donne lieu à aucune phénoménalisation possible, même si, comme le dit un étudiant, cette impossibilité même pousse à la transcendance.

«C'est le plus proche du vivant, une femme couchée à la vie. Les viscères parlent plus de la vie que la bidoche. Elle vivrait ce moment, elle vivrait sa mort. » (Xavier, G. 2001)

Planche 7 


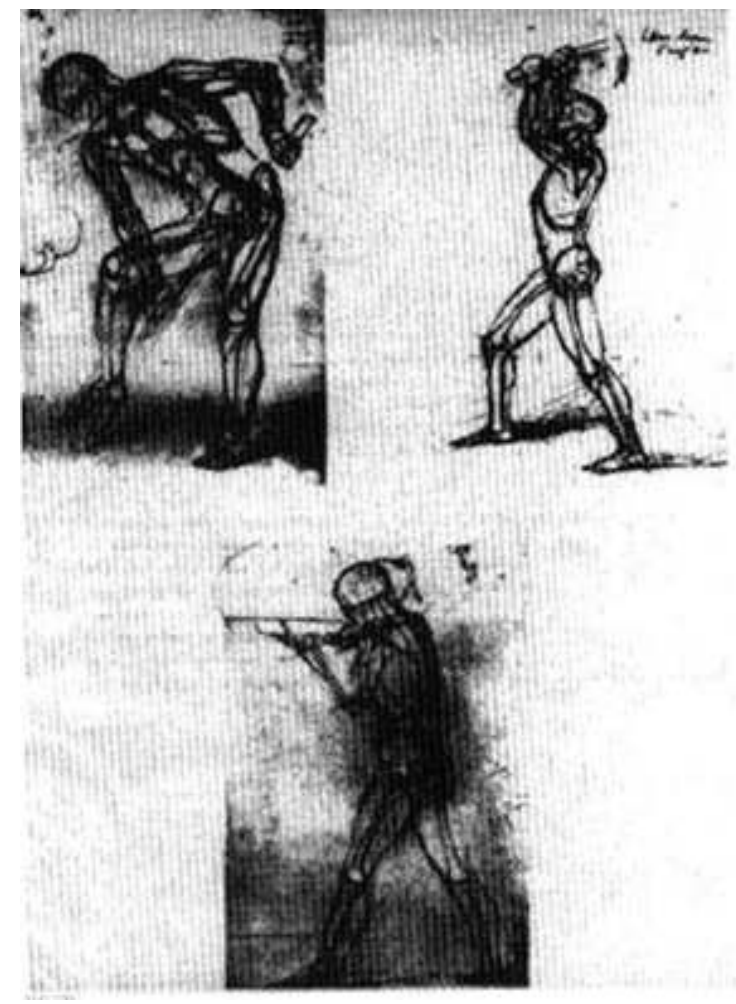

Planche 7. Anatomie artistique. Barcsay (Berger-Levrault, 1982)

Cette reproduction, tirée d'un traité d'anatomie artistique, pourrait sembler la plus proche des représentations de l'anatomie pour les étudiants. Il n'en est rien, car si l'aspect mécanique en est souligné, on regrette son manque de précision et de rigueur.

«Ça montre les mouvements. On a le sentiment de mouvement, donc de déplacement. Ça fait trop schéma, esquisse. » (Élisabeth, B. 2001)

126 Si le mouvement est présent, autant sous son aspect vital, quotidien, que sous l'angle de considérations mécaniques, c'est la structure du corps qui manque.

«Je sais pas, on voit mais c'est moins précis... Ils se servent toujours de l'anatomie comme support, mais c'est moins précis, moins exact... ça permet pas de comprendre le geste, c'est très flou. On voit pas, comme sur un squelette, tous les os du corps humain, c'est un peu flou. L'anatomie, c'est exact. » (Marie-Aude, L. 1991)

Planche 8 


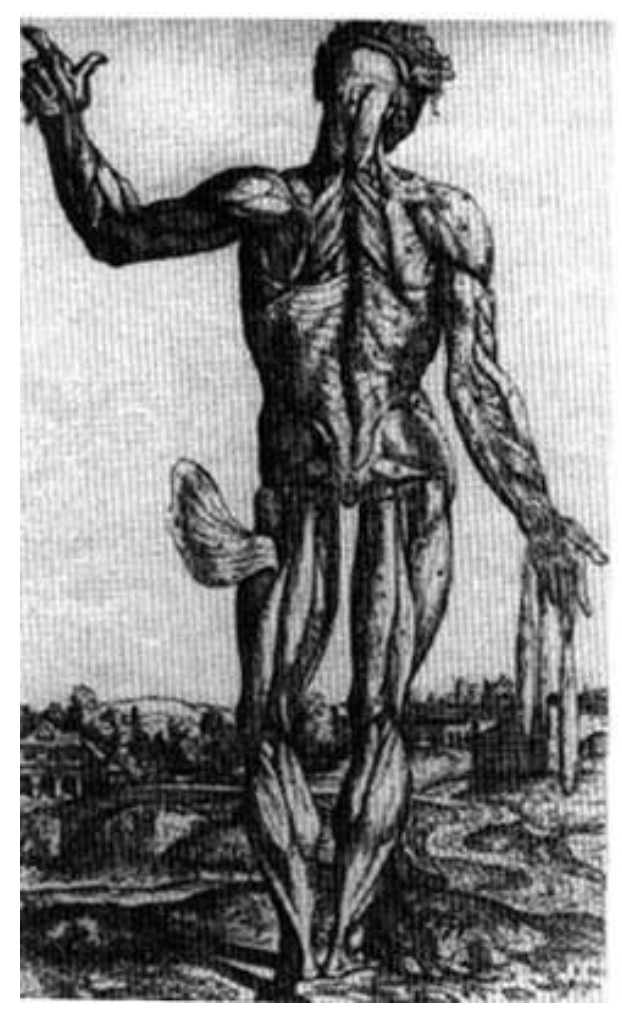

Planche 8. Iconographie anatomique André Vesale (Edition Dacosta, Paris, 1980)

Planche peu commentée, mais généralement appréciée comme figuration adéquate de l'anatomie, et qui résout le problème du rapport de la vie et du mort.

« On voit mieux, c'est plus précis. Il y a un grand nombre de muscles. C'est curieux,

il est debout, en mouvement. » (Cyril, R. 2001)

Les commentaires rappellent, dans leur spontanéité, les remarques développées par G. Canguilhem dans son analyse de l'œuvre de Vésale : «L'homme de Vésale reste un sujet responsable de ses attitudes. L'initiative de la posture selon laquelle il s'offre à l'examen lui appartient, et non au spectateur » (1983:31).

«Elle est marrante... parce qu'elle a des cheveux. Elle est presque vivante. Puis le contexte aussi. Il y a le pont, une rivière, des maisons. Ça fait un ensemble tout à fait vivant, je trouve.» (Corinne, P. 1991)

Si la planche 8 provoque des commentaires favorables, c'est parce qu'elle est congruente avec le corps anatomique que les étudiants se représentent. Le savoir anatomique n'est plus science, construction épistémique, mais image de l'Homme d'un Anthropos-, point de vue partiel qui n'abolit pas les jugements ontologiques, qui ne naturalise aucunement l'humain, mais lui laisse une identité biologique inscrite dans ses pratiques.

« Ça, ça me fait penser à l'anatomie parce qu'on voit des muscles, pareils, et là ça va de plus en plus vers le dessin et en plus, on voit l'homme, mais il est vivant. » (Stéphanie, D. 2001)

Pour conclure

Le contenu des entretiens, précisé par les commentaires relatifs aux planches, traduit une structure générale des représentations de l'anatomie chez les étudiants en STAPS. 
Celle-ci s'organise autour de quatre références qui donnent pour eux un sens à l'anatomie :

- une référence épistémique pour laquelle l'anatomie a les caractères d'une science positive et les critères d'une épistémê empiriquement validée;

- une référence à la matière, à la structure physique du corps telle qu'elle peut être donnée à voir;

- une référence pratique pour laquelle l'unité psychologique du moi cimente la valeur accordée au savoir anatomique dans un processus phénoménal qui relie la réification du corps à l'expérience du corps propre;

- une référence ontologique et métaphysique où se pose le rapport de la vie avec le mort dans l'optique d'un réalisme qui oscille entre matérialisme et concrétude factice, et le rapport du vivant avec la mort posé dans l'inquiétude d'un fatum (voir tableau).

L'anatomie de l'homme apparaît comme anthropologie, compréhension de l'humain, beaucoup plus que comme science naturaliste ou donnée biologique. On peut véritablement parler d'une anthropologisation de l'anatomie humaine par les étudiants... Processus quasi inverse de celui d'une constitution de l'anthropologie physique et positive. Il ne devient pas étonnant, en conséquence, que les étudiants établissent une partition entre l'anatomie et la biologie : cette dernière "s'est efforcée de se donner un vocabulaire tel que l'on puisse parler des vivants sans parler de la vie, sans faire appel à d'autres langues que celle du physicien ou du chimiste » (Canguilhem 1983 : 34), ce que l'anatomie ne permet pas. Car reste la figure du corps, qui n'est pas uniquement structure vivante, mais emblème de la vie.

Il est donc compréhensible que les étudiants développent une version romantique de l'anatomie, dans laquelle l'imagination et l'expérience constituent des modes de connaissance. Le corps anatomique est soumis à l'anagramme, une recomposition de ses significations, modulées sur les attentes et les impossibles (Bellmer 1977 : 197).

Le sport et les activités physiques constituent une culture du geste, non sur le mode de l'expressivité mais sur celui de la pragmatique, de la technique ${ }^{12}$. Existent conjointement un discours technique, qui est discours porté sur le geste, le mouvement, et un récit dont le sport dans son ensemble est le sujet. L'un et l'autre font référence à un corps articulaire tel que Guillemette Bolens (2000:216) le pose dans son analyse de la littérature occidentale. Elle souligne que ce corps articulaire, relié au sens $\mathrm{du}$ mouvement relève d'une mentalité orale qui est toujours plus active que contemplative. «Ce n'est pas le corps comme objet d'observation mais comme événement, comme système de jonction et de séparation, comme lieu de mobilité dont il s'agit, et il se dit dans un rapport aux sensations, aux émotions, aux idées qu'il occasionne ».

Une telle mentalité orale semble traverser le sport et marque les études en «Sciences et techniques des activités physiques et sportives ». On peut alors se demander si le langage scientifique ne prend pas là valeur de récit, d'un récit congruent avec le discours technique et au service de la constitution de l'Anthropos ${ }^{13}$. 


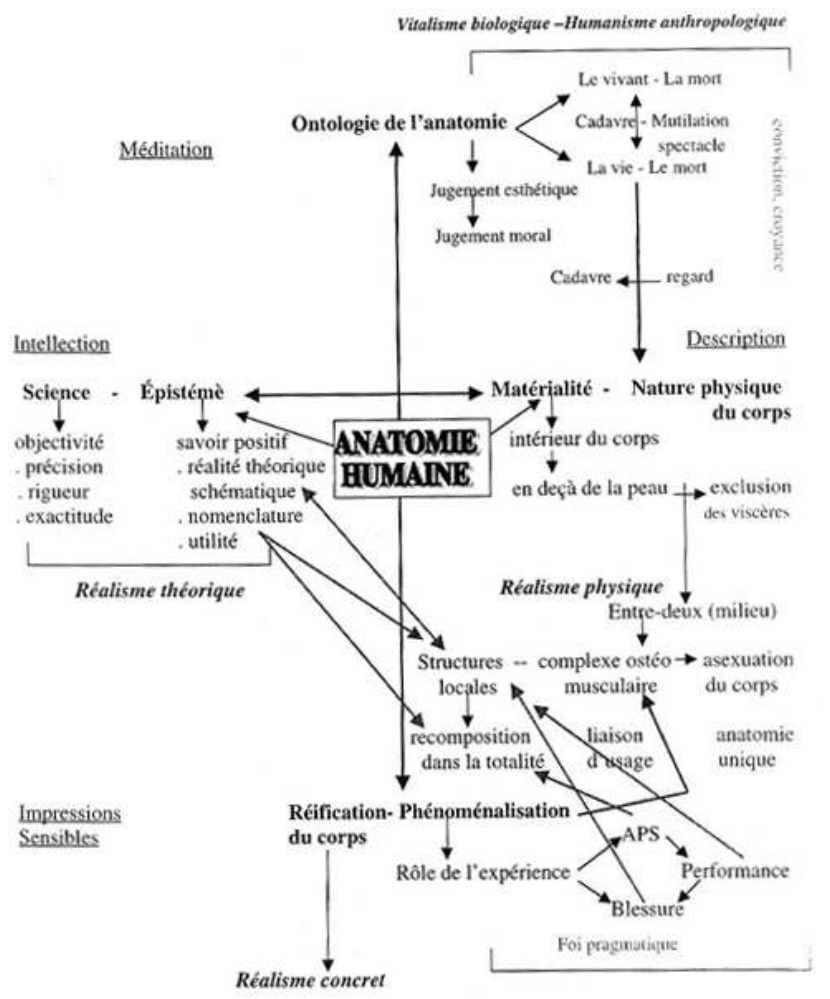

\section{BIBLIOGRAPHIE}

Bellmer, Hans

1977 Petite anatomie de l'inconscient physique ou l'anatomie de l'image. Paris : Éditions E. Losfeld, Le Terrain Vague.

Biache, Marie-Joseph

1996 «Qu'est-ce qu'un sport féminin ? Essai d'épistémologie appliquée. Le cas du hand-ball », in P. Arnaud et Th. Terret (eds), Histoire du sport féminin, tome 2. Paris : L'Harmattan.

Bolens, Guillemette

2000 La Logique du corps articulaire : les articulations du corps humain dans la littérature occidentale. Rennes : Presses Universitaires de Rennes.

Canguilhem, Georges

1968 « Machine et organisme », in La connaissance de la vie. Paris : Librairie Philosophique J. Vrin. 1983 « L'homme de Vesale dans le monde de Copernic : 1543 », in Études d'histoire et de philosophie des sciences. Paris : Librairie Philosophique J. Vrin (1ère édition 1968).

Dagognet, François

1987 Marey : la passion de la trace. Paris : Hazan éditeur. 


\author{
Descargues, Pierre \\ 1980 « Introduction », in Dessins d'art et traités d'anatomie. Paris : Société Nouvelle des Éditions du \\ Chêne. \\ Guillermé, Jacques \\ 1977 Préface à l'écorché. Catalogue de l'exposition réalisée par l'École Régionale des Beaux-Arts de \\ Rouen. Éditions Musée des Beaux-Arts de Rouen. \\ Mach, Ernst \\ 1984 Remarques préliminaires anti-métaphysiques. Cahiers du Musée National d'Art Moderne. Paris : \\ Éditions Centre Pompidou. \\ Pociello, Christian \\ 1999 La Science en mouvements. Etienne Marey et Georges Demeny, 1870-1920. Paris : Presses \\ Universitaires de France. \\ Schmitt, Jean-Claude \\ 1990 La raison des gestes dans l'occident médiéval. Paris : Éditions Gallimard («Bibliothèque des \\ Histoires »). \\ Vigarello, Georges \\ 1978 Le Corps redressé. Paris : Éditions J.-P. Delage.
}

\title{
NOTES
}

1. Étude locale, réalisée à l'UFR de STAPS (Sciences et Techniques des Activités Physiques et Sportives) de l'Université Blaise Pascal de Clermont-Ferrand.

2. Lors des centaines d'entretiens d'orientation effectués avec les lycéens aspirant à intégrer le cursus en STAPS, l'enseignement de l'anatomie, et plus largement de la biologie, est constamment légitimé par ceux-ci, ce qui n'est pas le cas des sciences humaines. Les formations en STAPS, outre les enseignements pratiques et techniques dans les activités physiques et sportives, se composent de divers cours relatifs aux bases scientifiques de l'analyse de ces activités, les cours se partageant de façon égale entre un appui sur des sciences biologiques et un recours aux sciences humaines.

3. Une telle conception est épistémologiquement naïve et rappelle certaines injonctions de Jacques Lordat sur la prééminence de l'anatomique sur le physiologique dans les disputes concernant la constitution de la physiologie au XIXe siècle ( $c f$. Canguilhem 1968).

4. L'entraînement sportif ou l'éducation physique constituent pourtant des modes d'intervention sur la matière corporelle, mais c'est par l'intermédiaire d'exercices que des modifications anatomiques sont produites. Elles sont alors principalement morphologiques, sauf dans des cas marginaux.

5. L'inclination est à l'origine possible de la curiosité. Il faudrait donc, pour intéresser aux choses de l'anatomie, les présenter dans leur nature, afin qu'elles rencontrent le goût de connaître. « Pour instruire les hommes, il faudrait les séduire par l'attrait du plaisir, et comment donner de l'agrément à l'image de la mort ? [...]. Il n'est qu'un seul moyen de rendre l'Anatomie intéressante et facile, c'est d'offrir les objets avec leurs couleurs naturelles. » (Gautier D’Agoty, Cours complet d'anatomie, 1773, cité par Guillermé 1977).

6. Les informateurs qui insistent sur l'utilité de la connaissance anatomique du rachis ont tous suivi des cours illustrés à l'aide d'un squelette véritable, contrairement aux 
étudiants actuels, pour lesquels les cours se rapportent presque exclusivement à des schémas partiels qui sont des images de préparations anatomiques. On peut ainsi percevoir une différence notable dans les représentations de l'utilité de l'anatomie : si pour les étudiants de 2001, l'intérêt prophylactique est avant tout local et attaché à la charge de l'exercice (ce sont les blessures musculaires et ligamentaires qui ont été citées), c'est toute l'architecture du squelette qui est mise en exergue par les étudiants de 1991, la blessure à prévoir et à éviter consistant en un désordre total de la structure. 7. Cette conception première, qui s'impose par la force de son réalisme est à l'origine de l'anatomie médicale. Elle est aussi dépassée depuis la révolution physiologique du XIXe siècle mais reste inscrite comme analogie fondamentale, façon de se repérer dans une réalité qui est celle d'un paysage, d'un espace. Façon de pérégriner. « Ainsi l'image de la réalité intérieure humaine ne diffère-t-elle pas d'une carte routière. Elle propose un schéma mental de fonctionnement. Elle est de même ordre que les vues cavalières où les archéologues rebâtissent en perspective les villes dont ils ne connaissant que les ruines... l'image anatomique n'offre pas une représentation du réel. Elle procure, dans l'analyse, l'occasion de se référer constamment à une synthèse, quelque chose comme le plan qui propose au voyageur, sur une route, des informations sur sa position, son environnement, les services qu'il peut attendre de la ville voisine, du réseau des télécommunications accessibles. »(Descargues $1980: 12$ ).

8. On peut repérer une forme naïve et métaphysique de la discussion épistémologique du rapport mouvement-mécanisme telle que l'expose G. Canguilhem. La conception « mécanique » de l'anatomie que les étudiants énoncent rapporte le corps à sa configuration; elle reste épistémologiquement indéterminée et s'inscrit dans un mécanisme candide fondé sur la simple analogie de structure. (cf. Canguilhem 1968). 9. Tel qu'il est employé par les étudiants, y compris en 2001, le terme de «biomécanique » recouvre la définition de la iatromécanique, et ne prend pas le sens que les travaux de J. E. Marey lui ont assigné (Dagognet 1978; Pociello 1999) et qui recouvre ce que nous nommons «mécanique fonctionnelle » afin d'éviter toute confusion.

10. «Autopsie, en effet, signifie voir par soi-même :j'ai vu, de mes yeux vu, ce qui est su ailleurs, ce qui est écrit dans le livre. Autopsier, c'est passer du savoir abstrait à la connaissance pratique ». (Descargues $1980: 12$ ).

11. Il semble que la présentation de la femme en termes de déficits structurel et fonctionnel soit une constante représentationnelle dans le discours sportif. La femme n'y est que rarement représentée en propre, mais toujours rapportée à l'homme. La figure virile est l'étalon de toute performance sportive. (cf. Biache 1996).

12. "Je soutiendrais que le Moyen-Âge est le berceau de notre conception technique des gestes du travail. Mais aussi que le faire le plus important pour la culture médiévale y est d'un tout autre ordre : celui de la croyance, fondement de l'efficacité symbolique des gestes rituels, magiques et sacramentels ». (Schmitt 1990). On peut, par hypothèse, trouver là l'explication d'une fonction contemporaine du sport qui réside dans le symbolique et la ritualisation.

13. Le terme d'éducation physique semble aussi obsolète que l'anatomie elle-même. L'action directe sur le corps, son redressement, n'est plus de mise dans les gymnastiques contemporaines qui, toutes, portent l'accent sur le mouvement et ses intérêts pour la personne (Vigarello 1978). 


\section{RÉSUMÉS}

L'enseignement de l'anatomie humaine est considéré par les étudiants en Sciences et Techniques des Activités Physiques et Sportives comme absolument indispensable. L'anatomie est posée comme science fondamentale car elle promeut un matérialisme ontologique et évacue apparemment tout questionnement psychologique ou philosophique. Cette étude, menée en deux périodes à dix ans d'intervalle auprès d'étudiants ayant suivi les enseignements d'anatomie descriptive et fonctionnelle, montre a contrario, que la réalité " corps anatomique » reste à leur yeux essentiellement phénoménale, liée à des représentations et croyances issues de leur expérience propre et dont l'expression trahit des métaphysiques concurrentes. On peut véritablement parler d'une anthropologisation de l'anatomie par les étudiants en STAPS.

The body and its anatomy. Representations and beliefs. Survey among STAPS students The students in Sciences and Techniques of Physical Activities and sports (staps) consider courses in human anatomy as absolutely essential. Anatomy is thought to be a fundamental topic in that it promotes an ontological materialism and apparently evacuates all psychological or philosophical questioning. The present study is based on two surveys carried out ten years apart among students having taken descriptive and functional anatomy lessons. It shows that the body stays essentially a phenomenal reality linked to representations and beliefs issued from the students' own experience, the expression of which reveals competing metaphysics. It is therefore possible to speak of an anthropologisation of anatomy by staps students.

El cuerpo y su anatomía. Investigaciones con estudiantes de Ciencias y Técnicas de las Actividades Físicas y Deportivas.

Los estudiantes de Ciencias y Técnicas de las Actividades Físicas y Deportivas consideran absolutamente indispensable el estudio de la anatomía humana. La anatomía se plantea como ciencia fundamental dado que promueve un materialismo ontológico y elimina aparentemente cualquier cuestionamiento psicológico o filosófico. Este estudio, que ha sido llevado a cabo entre estudiantes que han seguido estudios de anatomía descriptiva y funcional, a lo largo de dos periodos separados por un intervalo de diez años, muestra por el contrario que la realidad « cuerpo anatómico », en la perspectiva de los sujetos estudiados, continúa siendo esencialmente fenoménica, ligada a representaciones y creencias que nacen de su propia experiencia, y cuya expresión viene a revelar metafísicas concurrentes. En verdad se puede hablar de que los estudiantes de «staps » llevan a cabo una antropologización de la anatomía.

\section{INDEX}

Mots-clés : anatomie humaine, représentation, enseignement sportif, analyse de discours

\section{AUTEUR}

\section{MARIE-JOSEPH BIACHE}

Laboratoire d'Anthropologie des Pratiques Corporelles. UFR de STAPS. Université Blaise Pascal, Clermont-Ferrand. 